\title{
An Infrared Census of DUST in Nearby Galaxies with Spitzer (DUSTiNGS). V. The Period-Luminosity Relation for Dusty Metal-poor AGB Stars
}

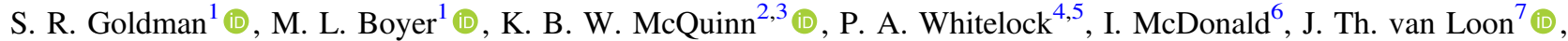 \\ E. D. Skillman ${ }^{8}$ (I) , R. D. Gehrz ${ }^{8}$ (1) A. Javadi ${ }^{9}$, G. C. Sloan $^{1,10}$, O. C. Jones ${ }^{11}$ (i), M. A. T. Groenewegen ${ }^{12}$, and J. W. Menzies ${ }^{4}$ \\ ${ }^{1}$ Space Telescope Science Institute, 3700 San Martin Drive, Baltimore, MD 21218, USA; sgoldman@ stsci.edu \\ ${ }^{2}$ University of Texas at Austin, McDonald Observatory, 2515 Speedway, Stop C1402, Austin, TX 78712 USA \\ ${ }^{3}$ Rutgers University, Department of Physics and Astronomy, 136 Frelinghuysen Road, Piscataway, NJ 08854, USA \\ ${ }_{5}^{4}$ South African Astronomical Observatory, P.O. Box 9, 7935 Observatory, South Africa \\ 5 Department of Astronomy, University of Cape Town, 7701 Rondebosch, South Africa \\ ${ }^{6}$ Jodrell Bank Centre for Astrophysics, Alan Turing Building, University of Manchester, M13 9PL, UK \\ ${ }^{7}$ Lennard-Jones Laboratories, Keele University, ST5 5BG, UK \\ ${ }^{8}$ Minnesota Institute for Astrophysics, School of Physics and Astronomy, 116 Church Street SE, University of Minnesota, Minneapolis, MN 55455, USA \\ ${ }^{9}$ School of Astronomy, Institute for Research in Fundamental Sciences (IPM), P.O. Box 19395-5531, Tehran, Iran \\ ${ }^{10}$ University of North Carolina Chapel Hill, Chapel Hill, NC 27599-3255, USA \\ ${ }^{11}$ UK Astronomy Technology Centre, Royal Observatory, Blackford Hill, Edinburgh EH9 3HJ, UK \\ ${ }^{12}$ Koninklijke Sterrenwacht van België, Ringlaan 3, B-1180 Brussels, Belgium \\ Received 2019 January 31; revised 2019 February 14; accepted 2019 February 18; published 2019 May 23
}

\begin{abstract}
The survey for DUST in Nearby Galaxies with Spitzer (DUSTiNGS) has identified hundreds of candidate dustproducing asymptotic giant branch (AGB) stars in several nearby metal-poor galaxies. We have obtained multiepoch follow-up observations for these candidates with the Spitzer Space Telescope and measured their infrared (IR) light curves. This has allowed us to confirm their AGB nature and investigate pulsation behavior at very low metallicity. We have obtained high-confidence pulsation periods for 88 sources in seven galaxies. We have confirmed DUSTiNGS variable star candidates with a $20 \%$ success rate and determined the pulsation properties of 19 sources already identified as thermally pulsing AGB stars. We find that the AGB pulsation properties are similar in all galaxies surveyed here, with no discernible difference between the DUSTiNGS galaxies (down to $1.4 \%$ solar metallicity; $[\mathrm{Fe} / \mathrm{H}]=-1.85$ ) and the far more metal-rich Magellanic Clouds (up to 50\% solar metallicity; $[\mathrm{Fe} / \mathrm{H}]=-0.38)$. These results strengthen the link between dust production and pulsation in AGB stars and establish the IR period-luminosity relation as a reliable tool $( \pm 4 \%)$ for determining distances to galaxies, regardless of metallicity.
\end{abstract}

Key words: galaxies: dwarf - galaxies: stellar content - infrared: stars - Local Group - stars: AGB and post-AGB stars: carbon

Supporting material: figure set, machine-readable tables

\section{Introduction}

Variable stars on the asymptotic giant branch (AGB) are known to show a linear correlation between the logarithm of the period and luminosity (Gerasimovič 1928). This relationship reveals details about the stellar physics that drives AGB evolution and, like the period-luminosity $(P-L)$ relationship for Cepheids and RR Lyrae, is a useful distance indicator. As such, the AGB $P-L$ relationship has been studied extensively over the years (Feast et al. 1989; Hughes \& Wood 1990; Ita et al. 2004a; Glass et al. 2009; Soszyński et al. 2009). Many of these studies focus only on Galactic and Magellanic AGB variables, since AGB stars are difficult to identify and resolve in more distant galaxies, mainly due to extinction by circumstellar dust that almost always accompanies large-amplitude pulsation. As a result, the properties of the AGB $P-L$ relation are not well constrained at low metallicity, and some uncertainty remains regarding its usefulness as a distance indicator in metal-poor galaxies and/or metal-poor galaxy halos (Feast et al. 2002).

Strong AGB pulsations and dust production are known to be tightly linked (Lagadec \& Zijlstra 2008; Sloan et al. 2016; McDonald et al. 2018; McDonald \& Trabucchi 2019). As a star evolves through the AGB phase, the strength of the pulsations grows, which simultaneously levitates more material to large circumstellar radii, where it condenses into dust. The AGB stars can be either oxygen-rich (M-type) or carbon-rich (carbon stars), producing silicate-rich and carbonaceous dust, respectively. The processes dictating the envelope chemistry are third dredge-up events (TDUs) and hot-bottom burning (HBB), which depend primarily on a star's initial mass (see the reviews by Herwig 2005; Karakas \& Lattanzio 2014) and metallicity. Carbon stars produce enough carbon internally that they can produce significant amounts of dust regardless of their initial metallicity (Sloan et al. 2012), while oxygen-rich stars require heavier elements (e.g., $\mathrm{Fe}, \mathrm{Mg}, \mathrm{Al}, \mathrm{Si}$ ), which must be produced by a previous generation of stars or the by-products of TDUs and HBB (e.g., Sloan et al. 2010; Bladh et al. 2015).

How AGB mass loss, dust production, and evolution are affected by metallicity is still unclear. Variability studies in the Large (LMC) and Small (SMC) Magellanic Clouds have produced large samples of long-period variables (LPVs), but these samples span a narrow range in metallicity. Additional works have discovered large samples of LPVs in globular clusters and smaller samples in nearby dwarf galaxies (see Section 1.3). Here we present the first large-scale IR survey of LPVs in nearby galaxies, reaching lower metallicity than ever before. 


\subsection{LPV Stars}

The driving force behind AGB pulsations is poorly understood. While sources within the instability strip of the Hertzsprung-Russell (H-R) diagram (e.g., Cepheid variables or RR Lyrae stars) pulsate as a result of a gravity-opacity instability known as the $\kappa$-mechanism, the large convection cells within an AGB star would likely disrupt both spherical symmetry and this mechanism (Liljegren et al. 2018).

Red giants and supergiants (RSGs) and AGB stars can follow several sequences on the $P-L$ diagram, often labeled A through E (Wood et al. 1999; Ita et al. 2004a). ${ }^{13}$ Many of the sources on these sequences pulsate in multiple modes, with secondary periods falling on the other sequences (see Trabucchi et al. 2018). The $B$ and $C^{\prime}$ sequences are composed of red giant branch (RGB) and AGB first-overtone radial pulsators. The dusty and evolved AGB stars, or Mira variables, primarily lie along the fundamental mode (sequence 1; Riebel et al. 2010), also known as sequence C (Wood et al. 1999); however, some $(\sim 30 \%)$ lie along sequence $\mathrm{D}$, with pulsation periods between 500 and 2000 days. These have been referred to as long secondary periods (LSPs) yet are clearly the dominant mode in some evolved stars (Nicholls et al. 2009; Trabucchi et al. 2017); the sequence will be discussed further in Section 5.5.

\subsection{Dust at Low Metallicity}

As a result of their different masses, AGB stars can have considerably different lifetimes. The main-sequence lifetime of AGB progenitors (low- and intermediate-mass stars; 0.8-8 $M_{\odot}$ ) is between 0.1 and $12 \mathrm{Gyr}$, after which they typically spend $20 \%$ of that time as red giants, $\sim 1 \%$ as early-AGB stars, and $\sim 0.1 \%$ as thermally pulsing AGB (TP-AGB) stars (Marigo et al. 2008, 2017; Javadi et al. 2011b, 2017). On the TP-AGB, these stars will produce the most dust and contribute the most mass back to the interstellar medium (ISM; see review by Höfner \& Olofsson 2018). Recent works exploring the metallicity dependence of dust production in carbon stars have produced mixed results. A strong dependence was originally suggested by van Loon (2000) and corroborated by van Loon et al. (2005) and van Loon (2006), while McDonald et al. (2011) and Sloan et al. $(2012,2016)$ found little to no dependence. Nanni et al. $(2013,2014)$ and Ferrarotti \& Gail (2006) gave estimates of the metallicity dependence of the dust production using theoretical models. Work within the Galaxy and the Magellanic Clouds has also allowed us to study the effect of metallicity on the mass loss of oxygen-rich AGB stars (van Loon 2000, 2006; Goldman et al. 2017), with the results showing little to no effect on the measured mass-loss rates.

The DUST in Nearby Galaxies with Spitzer survey (DUSTiNGS; Boyer et al. 2015b, hereafter Paper I) searched for dust-producing AGB stars in 50 nearby $(<1.5 \mathrm{Mpc})$ metalpoor $(-2.7<[\mathrm{Fe} / \mathrm{H}]<-1.0)$ dwarf galaxies using Spitzer Space Telescope (Werner et al. 2004; Gehrz et al. 2007) InfraRed Array Camera (IRAC; Fazio et al. 2004) channels 1 and 2. The survey discovered hundreds of candidate dustproducing AGB stars at metallicities as low as $0.6 \%$ solar and provided no evidence for a strong metallicity dependence in overall dust production (Boyer et al. 2015a, hereafter Paper II). Observations at wavelengths longer than $\lambda=5 \mu \mathrm{m}$, where thermal emission from circumstellar dust dominates the IR

\footnotetext{
13 These sequences have also been labeled as $1-4, \mathrm{D}$, and E (e.g., Riebel et al. 2010).
}

spectral energy distribution (SED), will be required to confirm this. Boyer et al. (2017, hereafter Paper IV) identified 146 carbon- and oxygen-rich stars by exploiting the strength of the water features in M-type stars and the $\mathrm{CN}+\mathrm{C}_{2}$ features found in carbon stars. Though most (120) of these sources were classified as carbon-rich, 26 were identified as M-type. These observations showed that dust is produced by both carbon- and oxygen-rich AGB stars over the full metallicity range spanned by DUSTiNGS. This suggests that metal-poor high-mass AGB stars can produce dust as early as $30 \mathrm{Myr}$ after forming (for a $10 M_{\odot}$ star), while lower-mass carbon stars form dust after roughly 0.3-3.6 Gyr (van Loon et al. 2005). Therefore, AGB stars are likely important contributors of dust in the early universe. This work also led to the discovery of a potential dust-producing super-AGB star in IC 10 with an assumed mass $\sim 8-12 M_{\odot}$ and strong water absorption indicative of an AGB star. Super-AGB stars are more massive $\left(6 M_{\odot} \lesssim M \lesssim 9 M_{\odot}\right)$ AGB stars that are capable of fusing carbon and developing a degenerate oxygen-neon core. There is evidence that they can be dusty (Javadi et al. 2013) and produce the ONeMg white dwarfs that are responsible for neon nova explosions (Evans \& Gehrz 2012). These stars may also be capable of ending in an electron-capture supernova without developing an iron core like the observationally similar RSGs (Doherty et al. 2015). We did not detect variability in this source due to a lack of temporal coverage (two epochs).

\subsection{Metal-poor LPV Samples}

Much of the information known about metal-poor LPVs is from the Optical Gravitational Lensing Experiment (OGLE; Udalski et al. 1997), Massive Compact Halo Object (MACHO; Alcock et al. 1997), and Spitzer Surveying the Agents of a Galaxy's Evolution (SAGE; Meixner et al. 2006; Riebel et al. 2010; Gordon et al. 2011; Riebel et al. 2015) surveys, with a handful of surveys in other galaxies (shown in Table 1). While a large number of metal-rich samples exists (Huang et al. 2018; Yuan et al. 2018), the majority of the more metal-poor LPVs have been found in Leo I, NGC 185, NGC 147, and NGC 6822, galaxies only slightly more metal-poor than the SMC. The most metal-poor sources to date were found by McDonald et al. (2010), who discovered two sequence-D variables in the globular cluster M15 at $[\mathrm{Fe} / \mathrm{H}]=-2.37$ dex (Harris 1996), and Whitelock et al. (2018), who discovered three LPVs in the Sagittarius dwarf irregular galaxy (Sag DIG). The metallicity of Sag DIG has been measured in both stars using red giants $\left([\mathrm{Fe} / \mathrm{H}]=-1.88_{-0.09}^{+0.13}\right.$; Kirby et al. 2017) and isochrones $([\mathrm{Fe} / \mathrm{H}]=-2.1$; Momany et al. 2002) and the gas $(12+\log$ $(\mathrm{O} / \mathrm{H})=7.26-7.50$; Skillman et al. 1989; Saviane et al. 2002). One of these LPVs in Sag DIG has a pulsation period of 950 days, indicating a very late stage of evolution, and was found to be oxygen-rich (Paper IV). Variables have also been detected in globular clusters (Clement et al. 2001; Feast et al. 2002; Lebzelter \& Wood 2005). However, their low mass limits these sources to the lower regions of the $P-L$ sequences.

It is difficult to study the $P-L$ relation at lower metallicities because so few LPVs have been discovered in this regime. The DUSTiNGS survey initially identified several LPV candidates using two-epoch photometry. Here we follow up with additional epochs and provide a larger sample to populate the $P-L$ diagram over a large metallicity range $(-1.27>[\mathrm{Fe} / \mathrm{H}]>-1.85)$. DUSTiNGS is the first large-scale IR survey to identify the dustiest evolved stars in these galaxies. These stars can be 
Table 1

Surveys of LPVs in Nearby Galaxies, Including This Work

\begin{tabular}{|c|c|c|c|c|c|c|c|}
\hline Galaxy & $\begin{array}{c}d \\
(\mathrm{Mpc})\end{array}$ & {$[\mathrm{Fe} / \mathrm{H}]$} & $\underset{(\mathrm{mag})}{12+\log (\mathrm{O} / \mathrm{H})}$ & $\begin{array}{c}M_{V} \\
(\mathrm{mag})\end{array}$ & $\begin{array}{l}\text { Number of Sources with } \\
\text { High-confidence Fit Periods }\end{array}$ & $\begin{array}{l}\text { Number from } \\
\text { Previous Work }\end{array}$ & Previous Work Reference \\
\hline And IX & 0.77 & $-2.20 \pm 0.20$ & $\cdots$ & $-8.1 \pm 1.1$ & 0 & $\cdots$ & \\
\hline DDO 216 & 0.92 & $-1.40 \pm 0.02$ & $7.93 \pm 0.13$ & $-12.2 \pm 0.2$ & 5 & $\ldots$ & \\
\hline Fornax $^{a}$ & 0.15 & $-0.99 \pm 0.01$ & $\ldots$ & $-13.4 \pm 0.3$ & $\ldots$ & 7 & Whitelock et al. (2009) \\
\hline IC 10 & 0.79 & -1.28 & $8.19 \pm 0.15$ & $-15.0 \pm 0.2$ & 16 & $\ldots$ & \\
\hline IC 1613 & 0.76 & $-1.6 \pm 0.20$ & $7.62 \pm 0.05$ & $-15.2 \pm 0.2$ & 15 & 9 & Menzies et al. (2015) \\
\hline Leo $I^{\mathrm{a}}$ & 0.25 & $-1.43 \pm 0.01$ & $\ldots$ & $-12.0 \pm 0.3$ & $\ldots$ & 26 & Menzies et al. (2010) \\
\hline NGC 147 & 0.68 & $-1.1 \pm 0.10$ & $\cdots$ & $-14.6 \pm 0.1$ & 8 & 168 & Lorenz et al. (2011) \\
\hline NGC 185 & 0.62 & $-1.3 \pm 0.10$ & $\ldots$ & $-14.8 \pm 0.1$ & 29 & 419 & Lorenz et al. (2011) \\
\hline NGC $6822^{\mathrm{a}}$ & 0.46 & $-1.0 \pm 0.5$ & $\ldots$ & $-15.2 \pm 0.2$ & $\cdots$ & $50+$ & Whitelock (2012) \\
\hline Phoenix $^{a}$ & 0.42 & $-1.37 \pm 0.2$ & $\cdots$ & $-9.9 \pm 0.4$ & $\cdots$ & 1 & Menzies et al. (2008) \\
\hline Sag DIG & 1.07 & $-2.1 \pm 0.20$ & $7.26-7.50$ & $-11.5 \pm 0.3$ & 0 & 3 & Whitelock et al. (2018) \\
\hline Sculptor ${ }^{\mathrm{a}}$ & 0.09 & $-1.68 \pm 0.01$ & $\ldots$ & $-11.1 \pm 0.5$ & $\ldots$ & 2 & Menzies et al. (2011) \\
\hline Sextans A & 1.43 & -1.85 & $7.54 \pm 0.06$ & $-14.3 \pm 0.1$ & 6 & $\cdots$ & \\
\hline Sextans B & 1.43 & -1.6 & $7.53 \pm 0.05$ & $-14.5 \pm 0.2$ & 0 & $\cdots$ & \\
\hline WLM & 0.93 & $-1.27 \pm 0.04$ & $7.83 \pm 0.06$ & $-14.2 \pm 0.1$ & 9 & $\cdots$ & \\
\hline
\end{tabular}

Note. Distances, $[\mathrm{Fe} / \mathrm{H}]$, and $M_{V}$ are from McConnachie (2012) and references therein; the metallicity for Sextans B is from Bellazzini et al. (2014). Kirby et al. (2017) also derived a higher metallicity for Sag DIG of $[\mathrm{Fe} / \mathrm{H}]=-1.88_{-0.09}^{+0.13}$, based on RGB star spectroscopy. The ISM gas-phase oxygen abundances $(12+\log (\mathrm{O} / \mathrm{H}))$ are from Mateo (1998), Lee et al. (2006), and Saviane et al. (2002). An alternative name for DDO 216 is Pegasus dwarf irregular.

${ }^{\text {a }}$ Galaxies not analyzed in this work.

obscured in the near-IR and optical. Observing in the IR ensures that all of the prominent dust producers are detected.

\section{Data and Observations}

We construct light curves using 3.6 and $4.5 \mu \mathrm{m}$ imaging data from the IRAC on board Spitzer, with a mix of programs from both the cryogenic and post-cryogenic phases. Data include new and archival observations from 2003 to 2017.

\subsection{DUSTiNGS}

The DUSTiNGS data include both the original Cycle 8 data obtained in 2011-2012 (PID: 80063) and data obtained during the Cycle 11 follow-up program (PID: 11041) in 2015-2016. Light curves are sparsely sampled owing to the spacing of the Spitzer visibility windows for the DUSTiNGS galaxies, which are roughly 4-6 weeks long. There are typically two windows each year, separated by approximately 6 months. The Cycle 8 program, described in Paper I, obtained two epochs, one in each visibility window. The Cycle 11 program obtained six additional epochs, with a pair of observations at the beginning and end of each of the three consecutive visibility windows. The cadence is illustrated in Figure 1.

\subsection{Archival Data}

By taking advantage of the rich archival history of the Spitzer Space Telescope, we have been able to use data from 11 observing programs (Table 2). Most of the archival data that we use here are from two programs.

SPIRITS - The SPitzer InfraRed Intensive Transients Survey (SPIRITS; Kasliwal et al. 2017) program (Cycles 10-12, PID: 10136, 11063) was aimed at discovering explosive transients, eruptive variables, and new IR events lacking optical counterparts. The SPIRITS targets include a few of the DUSTiNGs galaxies: IC 1613, NGC 147, NGC 185, Sextans A, and Sextans B. These observations were taken between the original DUSTiNGS epochs and the follow-up DUSTiNGS observations, filling a gap in our temporal coverage (Figure 1).

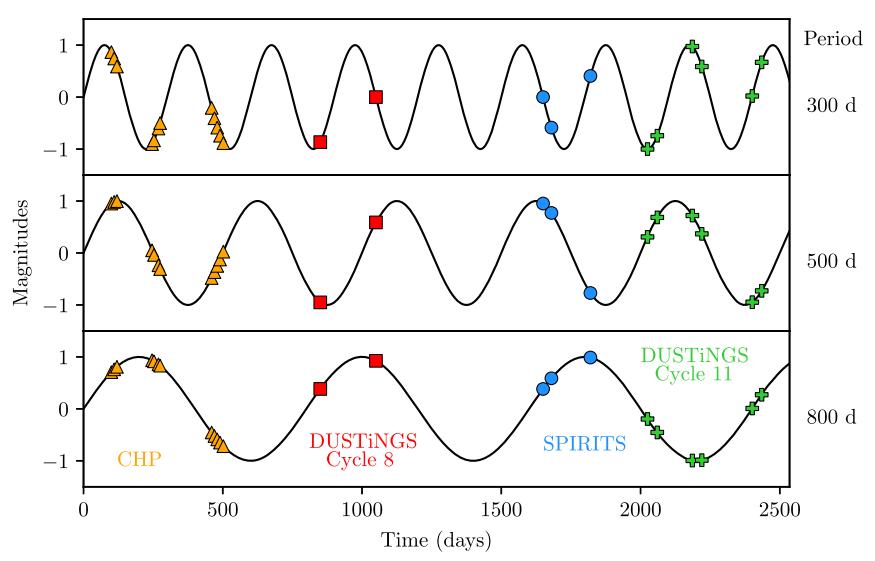

Figure 1. Artificial light curve showing the two initial DUSTiNGS observations (red) and six follow-up observations (green) fit with pulsation periods of 300, 500, and 800 days. Also shown is the cadence of the SPIRITS (blue) and CHP (orange) surveys; the cadence of the remaining archival programs is shown in Table 2.

Additional SPIRITS epochs cover the same epochs covered by the DUSTiNGS Cycle 11 observations and are included here.

The Carnegie Hubble Program-The Carnegie Hubble Program (CHP; Cycle 6, PID: 61001; Freedman et al. 2011) was aimed at determining distances to nearby galaxies using Cepheid variables. The CHP observations, taken between 2009 July and 2010 March, preceded both the DUSTiNGS and SPIRITS observations. The time between each epoch is $\sim 10$ days, given the focus on short-period variability (Figure 1).

We also use data from eight additional programs (individual observations are listed in Table 2) that sporadically sample the light curves between CHP and DUSTiNGS. The observations target 10 galaxies (listed in Table 1) that span a range in size $\left(-8.1 \mathrm{mag}>M_{V}>-15.2 \mathrm{mag}\right)$, distance $(0.62-1.43 \mathrm{Mpc})$, and, most notably, metal content $(-1.27>[\mathrm{Fe} / \mathrm{H}]>-1.85)$. We will use this range in metallicity to investigate its effect on the pulsation properties of evolved stars. 
Table 2

The Archival Spitzer Observations

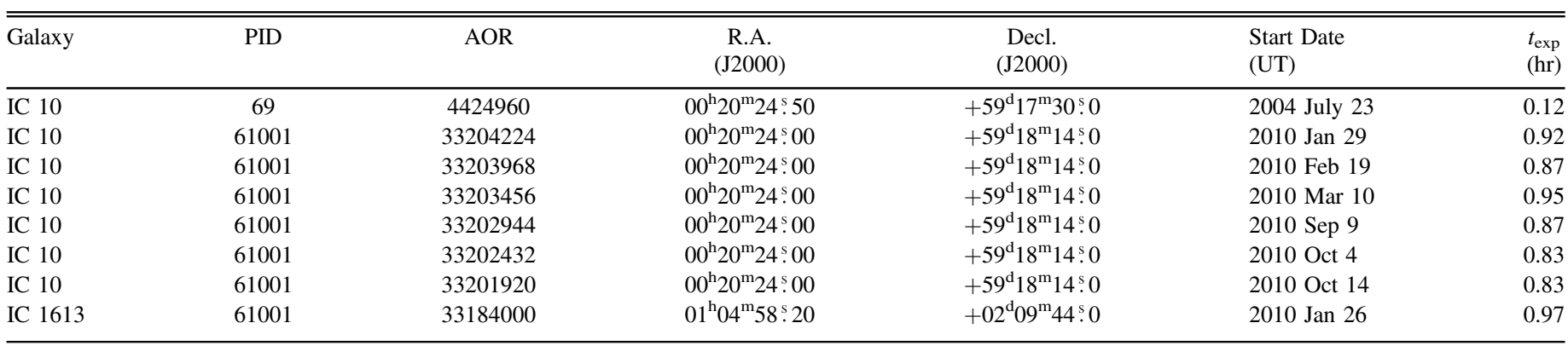

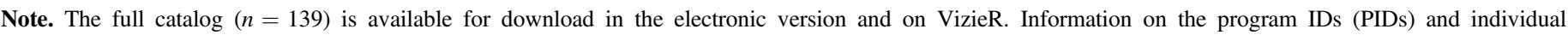
observations (AORs) can be found through the Spitzer Heritage Archive.

(This table is available in its entirety in machine-readable form.)

\section{Methods}

\subsection{PSF Photometry}

We have point-spread function (PSF) photometry on all of the DUSTiNGS sources and archival data using the DUSTiNGS pipeline (described in Paper I). We performed PSF photometry using DAOphot II and ALLSTAR (Stetson 1987) on the coadded frames for the fainter sources ([3.6] > $16 \mathrm{mag}$ ) and individual frames for the brighter sources. For the fainter sources, mosaicked images were used to reduce Eddington bias (Eddington 1913) in sources near the detection threshold. A mosaicked and subsampled image can smear the PSF, for example, if it includes a rotation between frames. As a result, single frames were used for the brighter sources, which are more sensitive to changes in the PSF. For the photometry of our sample in IC 10, we have adjusted the magnitudes by $0.2 \mathrm{mag}$ to account for foreground interstellar extinction (described further in Section 5.2). Paper I provides additional details on the photometry, saturation limits, photometric correction, and photometric completeness.

\subsection{Identifying $L P V s$}

The nonuniformity of the observing programs has resulted in varying depths and spatial coverage for each epoch. Therefore, many light curves are sparsely sampled. We have implemented the Lomb-Scargle algorithm (Lomb 1976; Scargle 1982) to determine the pulsation periods and amplitudes. The method fits a simple single-term sinusoidal light curve to different frequencies and then normalizes the results using the residuals. This method reduces the effects of unevenly spaced data using a more appropriate means of weighting within the Fourier transform (see review by VanderPlas 2018).

The nonuniform temporal and spatial coverage of the archival data has resulted in many sources with incomplete light curves from which we cannot derive reliable periods. To identify sources with sufficiently sampled light curves, we start by calculating the variability index (e.g., Gallart et al. 2004) that is defined as the ratio of the standard deviation of the measurements for a given star to the mean internal photometric uncertainty. A value of 1,2 , or 3 indicates variability at the $1 \sigma, 2 \sigma$, or $3 \sigma$ levels. We first restrict our light-curve fitting to stars with variability index $>1$. Second, we exclude sources with $<6$ epochs from our light-curve analysis, a number that was also concluded as sufficient by Javadi et al. (2015). Finally, we restrict light-curve fitting to sources brighter than $M_{[3.6]}=-7.5 \mathrm{mag}$, which includes all extreme
AGB (x-AGB) stars (Blum et al. 2006) in the LMC sample from Riebel et al. (2010). The x-AGB stars are the dustiest AGB stars that are likely in the superwind phase and very close to the end of their evolution. By restricting our sample to stars in the same brightness range as the LMC x-AGB stars, we limit contamination from fainter variable dusty objects, such as young stellar objects (YSOs) and background active galactic nuclei (AGNs).

\subsection{Light-curve Analysis}

For the sources that were included in the light-curve fitting, frequencies corresponding to 100-2000 days were fitted to each of the light curves using the Lomb-Scargle periodogram. Sources that were considered for further analysis were those that fit the following criteria.

1. A [3.6]-[4.5] color, of which the standard deviation did not deviate by more than $50 \%$ to eliminate sources that were not clearly dusty ${ }^{14}$ across epochs.

2. A best-fit solution where the peak frequency within the Fourier power spectrum was more than $6 \%$ higher than any other peak.

3. A best-fit solution constrained within a $95 \%$ confidence interval.

These three criteria determine which stars are included in further analysis. Stars excluded by these criteria are more likely to suffer from aliasing or poor data quality. The quality of the remaining sources was determined visually, with an eye for ensuring that the direction of brightness changes in the light curves matched the best-fit light curve, especially for shortterm changes. The results of the light-curve fitting are shown in Table 3, which includes the midline magnitudes, fit periods and amplitudes for both 3.6 and $4.5 \mu \mathrm{m}$ data, color properties, and classification confidence (described further below). An initial inspection of stars that pass these three criteria indicate that, at these distances, we are only able to reliably determine the pulsation periods of the dusty evolved sources using Spitzer.

Variations in spatial coverage between epochs caused some stars to be observed in only one of the filters, either [3.6] or [4.5]. Some of these light curves can be augmented where [3.6] data do not exist by including the $4.5 \mu \mathrm{m}$ data and using the mean color to derive $3.6 \mu \mathrm{m}$ magnitudes. This was done only if the color was determined to be stable $\left(\sigma_{[3.6]-[4.5]}<20 \%\right)$ and

\footnotetext{
${ }^{14}$ Sources that lacked $4.5 \mu \mathrm{m}$ measurements were still included in the final categorization and analysis.
} 
Table 3

The Results of 3.6 and $4.5 \mu \mathrm{m}$ Light-curve Fitting

\begin{tabular}{|c|c|c|c|c|c|c|c|c|c|c|c|c|c|}
\hline $\begin{array}{l}\text { (1) } \\
\text { Galaxy }\end{array}$ & $\begin{array}{c}(2) \\
\text { Target } \\
\text { ID }\end{array}$ & $\begin{array}{l}(3) \\
\text { R.A. } \\
\text { (deg) }\end{array}$ & $\begin{array}{l}\text { (4) } \\
\text { Decl. } \\
(\operatorname{deg})\end{array}$ & $\begin{array}{c}\text { (5) } \\
\langle 3.6 \mu \mathrm{m}\rangle \\
(\mathrm{mag})\end{array}$ & $\begin{array}{c}(6) \\
\langle 4.5 \mu \mathrm{m}\rangle \\
(\mathrm{mag})\end{array}$ & $\begin{array}{c}(7) \\
P \\
\text { (days) }\end{array}$ & $\begin{array}{c}(8) \\
P[4.5] \\
\text { (days) }\end{array}$ & $\begin{array}{c}\text { (9) } \\
\text { 2nd Fit } \\
\text { (days) }\end{array}$ & $\begin{array}{c}(10) \\
2 \text { nd [4.5] } \\
\text { (days) }\end{array}$ & $\begin{array}{l}(11) \\
\text { Amp. } \\
\text { (mag) }\end{array}$ & $\begin{array}{l}(12) \\
\text { Color } \\
(\mathrm{mag})\end{array}$ & $\begin{array}{c}(13) \\
\sigma_{[3.6]-[4.5]} \\
(\%)\end{array}$ & $\begin{array}{l}(14) \\
\text { Flag }\end{array}$ \\
\hline DDO 216 & 45582 & 352.2400208 & 14.61585045 & 16.65 & 16.46 & 101 & $\ldots$ & 131 & $\ldots$ & 0.35 & $\ldots$ & ... & IE \\
\hline DDO 216 & 58694 & 352.2214661 & 14.70353794 & 15.80 & 15.07 & 389 & 389 & 482 & 354 & 1.05 & 0.70 & 13.5 & RF \\
\hline DDO 216 & 76368 & 352.1983032 & 14.75455666 & 16.98 & 16.06 & 1308 & 196 & 223 & 178 & 0.34 & 0.92 & 16.2 & IE \\
\hline DDO 216 & 77533 & 352.1968384 & 14.73819065 & 17.04 & 15.91 & 103 & 174 & 110 & 1008 & 0.23 & 1.14 & 13.9 & UF \\
\hline DDO 216 & 83518 & 352.1894836 & 14.68728733 & 16.11 & 15.62 & 212 & 212 & 1339 & 1339 & 0.48 & 0.52 & 11.8 & UF \\
\hline
\end{tabular}

Note. Column 2 lists the DUSTiNGS IDs from Paper II, except for IDs over 5 million, which are new in this work. Columns 5 and 6 list the midline 3.6 and $4.5 \mu$ m magnitudes taken from the midline values of the best-fit light curve, columns 7 and 9 show the best- and second-best-fit pulsation periods ( $P$, 2nd fit), columns 8 and 10 list the same values for the $4.5 \mu \mathrm{m}$ fit, column 11 lists the fitted peak-to-peak $3.6 \mu \mathrm{m}$ amplitude, columns 12 and 13 list the median and standard deviation of the [3.6]-[4.5] color, and column 14 lists a quality flag for a high-confidence reliable fit (RF), insufficient epochs (IE), an unreliable fit (UF), or an LPV 5000+ (see Section 5.4). The full catalog $(n=261)$ is available in the electronic version and on VizieR.

(This table is available in its entirety in machine-readable form.)

had at least three epochs with color values. We refer to these photometric points as "simulated" photometry and show them in our light curves as empty circles (Appendix A, Figure 9). ${ }^{15} \mathrm{We}$ have similarly fitted the light curves of any sources with at least six epochs of $4.5 \mu \mathrm{m}$ data using simulated $4.5 \mu \mathrm{m}$ photometric values when possible. Only one source (IC 10 57276) did not have enough epochs to fulfill this requirement. These data are less sensitive (and therefore noisier), so we include them in Table 3 but use only the [3.6] data for further classification. For $65 \%$ of the full sample, the 3.6 and $4.5 \mu \mathrm{m}$ periods are the same. For $87 \%$ of the sample, the periods agree to within $10 \%$. For the remaining 11 sources, the standard deviation of the [3.6]-[4.5] color is large, and there is a difference in the number of epochs for all but two of the sources.

\subsection{Light-curve Categorization}

The light curves that pass the three criteria listed in the previous section are further categorized based on our confidence in the fits. These fall into four categories, two each considered "high-confidence" and "low-confidence." These groups are discussed further in Sections 4.1 and 4.2; the phased and unphased light curves of the high-confidence variables are shown in Appendix A.

High-confidence variables are as follows.

1. Reliable-fit (RF) sources with light curves that pass a visual inspection, meant to isolate sources with unique fit solutions.

2. LPV 5000+ sources that do not necessarily have RFs but are clearly variable on long timescales; described further in Section 5.4.

Low-confidence variables are as follows.

1. Insufficient epoch (IE) variable sources with RFs but where the uniqueness of the fit is unclear.

2. Unreliable-fit (UF) variable sources that do not pass visual inspection.

Recall that both the high- and low-confidence variables have a $\chi^{2}$ of at least $95 \%$ (Section 3.3), yet additional information about the uniqueness or quality of the fit is taken into account. The sources categorized as IEs are sources where a unique fit

\footnotetext{
$\overline{15}$ The displayed errors for the simulated photometry include the $\sigma_{[3.6]-[4.5]}$ and $4.5 \mu \mathrm{m}$ photometric uncertainties.
}

solution to the unphased light curve is not visibly clear. This may include a light curve lacking temporal coverage toward the maximum or minimum of the light curve, or where a shorter pulsation period could plausibly fit the source. A source designated as a UF is typically one that has a change in brightness in several epochs that is in the opposite direction of the change in the best-fit unphased light curve, outside the value of the uncertainty. Figure 12 in Appendix B shows examples of IE and UF sources.

We show the average [3.6]-[4.5] versus absolute $3.6 \mu \mathrm{m}$ magnitude color-magnitude diagram (CMD) of both high- and low-confidence sources in Figure 2. It is clear that we are only sensitive to the highly evolved and dusty $\mathrm{x}$-AGB stars here, as relatively dust-free $\mathrm{C}$-AGB and $\mathrm{O}-\mathrm{AGB}$ stars have smaller amplitude pulsations closer to the level of our photometric uncertainty (Riebel et al. 2015). We are also less sensitive to shorter-period variables due to our observing cadence. As a result, we do not obtain any high-confidence variables with median [3.6]-[4.5] colors less than $0.1 \mathrm{mag}$, and most of our measured pulsation periods are longer than 200 days.

Our final results include 92 high-confidence variables with four LPV 5000+ and 88 RF sources with a median period of 437 days. Figure 3 shows the variability of our low- and highconfidence variables. Note that UF sources cluster toward a low-variability index. We categorize the LPV 5000+ sources as high-confidence variables, but with a limited temporal baseline, we cannot definitively confirm their periodicity. The light curves of the low- and high-confidence variables are available for download in the electronic version and on VizieR.

\subsection{High-confidence Variables}

Figure 2 shows that the high-confidence variables occupy the same space as the $\mathrm{x}$-AGB sources found in the SAGE program (Riebel et al. 2010). The RF sample has a median $3.6 \mu \mathrm{m}$ absolute magnitude of $-9.2 \pm 0.26(1 \sigma)$. Four high-confidence variables have IR colors much redder than the rest of the sample ([3.6]-[4.5] > 1): IC 10 98211, IC 10 105991, NGC 18590369 , and WLM 84699. Their light curves, as well as the rest of the RF sources, are shown in Figure 9. We were not able to detect any RFs in And IX, Sextans B, or Sag DIG. This is not surprising, given that And IX and Sag DIG have limited temporal coverage and a small AGB population. 


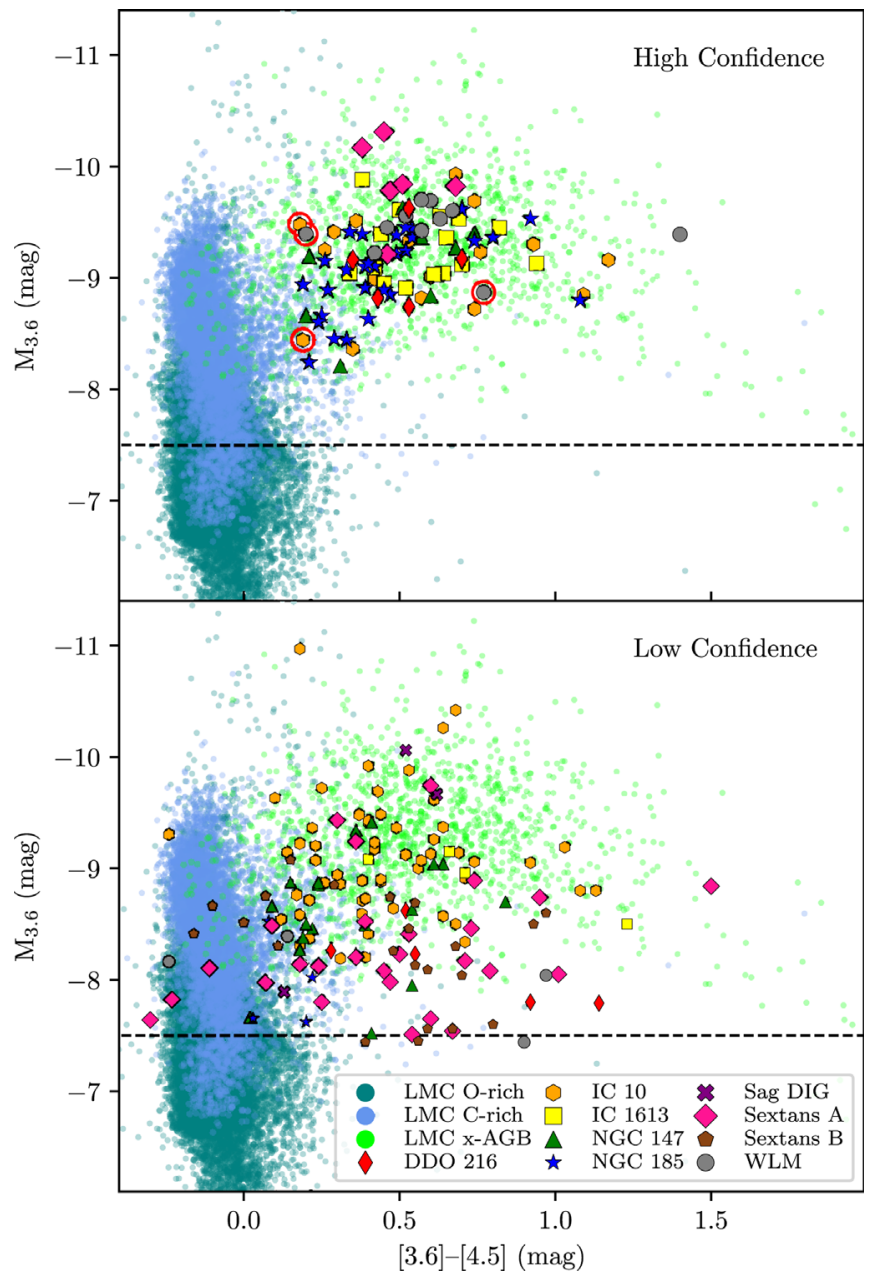

Figure 2. Average Spitzer IRAC [3.6]-[4.5] vs. $3.6 \mu \mathrm{m}$ absolute magnitude for the high- (top) and low-confidence (bottom) DUSTiNGS sources compared to the LMC sample from Riebel et al. (2010). Here $M_{3.6}$ was calculated using the midline value of the best-fit light curve. The brightness threshold for our lightcurve fitting analysis is shown with the dotted line. The LPV5000+ sources (described in Section 3.3) are shown with red circles. Adopted distances to the DUSTiNGS galaxies are shown in Table 1. For the LMC, we adopt a distance modulus of $M-m=18.52$ mag (Kovács 2000).

\subsection{Low-confidence Variables}

The low-confidence sample is composed of 113 sources with IEs and 56 sources with UFs. The low-confidence variables have a median absolute magnitude of $-8.55 \pm 0.17(1 \sigma)$, which is low compared to most of the LMC X-AGB sample. Sextans B has 14 sources with IEs and 12 sources with UFs. This may be the result of stochastic sampling. At fainter magnitudes, the photometric uncertainty is higher, making it more difficult to detect changes in brightness. This likely indicates that some sources in the lowconfidence sample are not LPVs but in fact YSOs and AGNs, which sometimes show irregular variability in the IR. We expect that low-confidence sources that cluster together may be YSOs in a star formation region. Sources far from the galaxy center may be AGNs. However, given the nonuniform positioning of the detectors and our sporadic temporal coverage, these sources are hard to disentangle (Paper I).

\subsection{Detection Statistics}

Of the sources that were included in our light-curve analysis, we have isolated a small subset as potential AGB stars from their

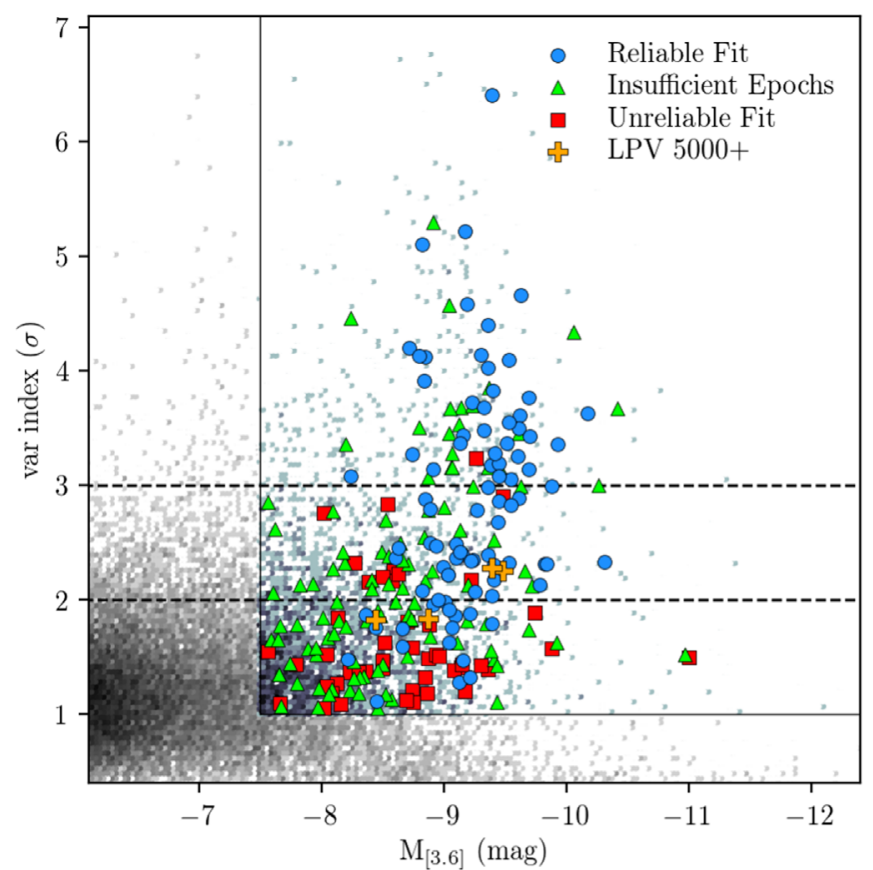

Figure 3. Standard deviation of the $3.6 \mu \mathrm{m}$ magnitude of the light curve divided by the average photometric uncertainty (variability index) vs. absolute $3.6 \mu \mathrm{m}$ magnitude for the DUSTiNGS sources with fitted light curves. Small points are those that did not meet the requirements of our light-curve fitting analysis (gray) and those that met the criteria but were not considered credible enough for further analysis (bluish). The $2 \sigma$ and $3 \sigma$ intervals are shown with dotted lines, and the designation of "LPV 5000+" is described further in Section 5.4.

Table 4

Results of Light-curve Fitting

\begin{tabular}{lccccc}
\hline \hline Galaxy & $\begin{array}{c}\text { Initial } \\
\text { Sample }\end{array}$ & $\begin{array}{c}\text { Var. } \\
\text { Index }>2\end{array}$ & $\begin{array}{c}\text { Dusty and } \\
\text { Variable }\end{array}$ & $\begin{array}{c}\text { Low } \\
\text { Conf. }\end{array}$ & $\begin{array}{c}\text { High } \\
\text { Conf. }\end{array}$ \\
\hline And IX & 16 & 2 & 1 & 0 & 0 \\
DDO 216 & 24,688 & 2166 & 15 & 7 & 5 \\
IC 10 & 80,351 & 5555 & 228 & 59 & 18 \\
IC 1613 & 311 & 96 & 27 & 5 & 15 \\
NGC 147 & 60,446 & 3712 & 70 & 20 & 8 \\
NGC 185 & 53,011 & 3506 & 58 & 4 & 29 \\
Sag DIG & 51 & 10 & 8 & 3 & 0 \\
Sextans A & 22,946 & 2959 & 83 & 40 & 6 \\
Sextans B & 22,629 & 2281 & 89 & 26 & 0 \\
WLM & 33,184 & 2860 & 34 & 5 & 11 \\
Total & 308,083 & 24,160 & 613 & 169 & 92 \\
\hline
\end{tabular}

Note. The table lists the number of high- and low-confidence variables per galaxy. Also shown is the number of sources and the number of sources that we determined were variable (variability index $>2$ ) and dusty and variable with [3.6]-[4.5]>0.2 mag and a variation in the color of less than $20 \%$.

[3.6]-[4.5] color and variability alone. These sources have already been limited to those that have at least six epochs and are bright in the IR $\left(M_{[3.6]}<-7.5 \mathrm{mag}\right)$. Table 4 shows the number of variable sources (var. index $>2$ ) in this sample and the number of sources that were dusty ([3.6]-[4.5] > 0.2) and also variable. Many of the sources that we were not able to confirm as AGB stars may be less luminous and dusty. These may have been confirmed as AGB stars given more observations.

In Paper II, we showed that our photometry is sensitive enough to detect variability down to peak-to-peak amplitudes of $\sim 0.15$ mag. We have now compared the number of sources with 
Table 5

Comparison to Literature Periods

\begin{tabular}{|c|c|c|c|c|c|c|c|c|}
\hline Galaxy & ID & $\mathrm{ID}_{\text {Lit. }}$ & $\begin{array}{l}\text { R.A. } \\
\text { (deg) }\end{array}$ & $\begin{array}{l}\text { Decl. } \\
\text { (deg) }\end{array}$ & $\begin{array}{c}P \\
\text { (days) }\end{array}$ & $\begin{array}{c}P_{\text {Lit. }} \\
\text { (days) }\end{array}$ & Type & Flag \\
\hline IC 1613 & 95038 & 1093 & 16.24090 & 2.15469 & 318 & 305 & $\mathrm{C}$ & $\overline{\mathrm{RF}}$ \\
\hline IC 1613 & 142830 & 3198 & 16.18219 & 2.05673 & 395 & 370 & $\mathrm{C}$ & $\mathrm{RF}$ \\
\hline NGC 147 & 68407 & 112 & 8.35656 & 48.55440 & 449 & 406 & $\ldots$ & RF \\
\hline NGC 147 & 112918 & 171 & 8.27302 & 48.47706 & 385 & $\ldots$ & $\mathrm{C}$ & RF \\
\hline NGC 147 & 113288 & 158 & 8.27241 & 48.50505 & 317 & 226 & $\mathrm{C}$ & IE \\
\hline NGC 147 & 123715 & 161 & 8.25264 & 48.46111 & 335 & 371 & $\ldots$ & UF \\
\hline NGC 185 & 70862 & 009 & 9.79879 & 48.32867 & 196 & 277 & $\mathrm{C}$ & RF \\
\hline NGC 185 & 77053 & 313 & 9.78671 & 48.35519 & 418 & 519 & $\ldots$ & RF \\
\hline NGC 185 & 83286 & 049 & 9.77481 & 48.38233 & 416 & 399 & M & $\mathrm{RF}$ \\
\hline NGC 185 & 87065 & 062 & 9.76783 & 48.36058 & 226 & 219 & $\ldots$ & RF \\
\hline NGC 185 & 87213 & 398 & 9.76754 & 48.35008 & 837 & 427 & $\ldots$ & RF \\
\hline NGC 185 & 89650 & 384 & 9.76315 & 48.32895 & 637 & 82 & $\mathrm{~S}$ & RF \\
\hline NGC 185 & 91361 & 076 & 9.76007 & 48.31672 & 360 & 358 & $\mathrm{C}$ & $\mathrm{RF}$ \\
\hline NGC 185 & 92015 & 078 & 9.75888 & 48.32191 & 416 & 420 & $\mathrm{~S}$ & RF \\
\hline NGC 185 & 95982 & 404 & 9.75180 & 48.32625 & 381 & 367 & $\ldots$ & $\mathrm{RF}$ \\
\hline NGC 185 & 96014 & 099 & 9.75173 & 48.32416 & 327 & 287 & $\mathrm{C}$ & $\mathrm{RF}$ \\
\hline NGC 185 & 131142 & 249 & 9.68842 & 48.33260 & 227 & 231 & $\cdots$ & $\mathrm{RF}$ \\
\hline NGC 185 & 136723 & 160 & 9.67694 & 48.31031 & 347 & 361 & $\mathrm{C}$ & $\mathrm{RF}$ \\
\hline NGC 185 & 132331 & 396 & 9.68612 & 48.35306 & 227 & 234 & $\mathrm{C}$ & UF \\
\hline
\end{tabular}

Note. Literature values (Lit.) are from Menzies et al. (2015) and Lorenz et al. (2011), where pulsation periods were derived using near-IR photometry (JHK).

high-confidence RFs that were covered in each epoch of the Cycle 11 observations with the number of $3 \sigma \mathrm{x}$-AGB stars from Paper II also found in that region (Appendix C). Of the $3 \sigma$ variables originally detected in Paper II and categorized as $\mathrm{x}$-AGB stars, we have confirmed $19 \%$ as RFs and likely TP-AGB stars. The remaining Paper II variables are outside of our spatial footprints and/or have temporal coverage that is too sporadic to measure a reliable light curve. The variable star catalogs presented here should therefore be considered a representative subset of the total variable population in each galaxy.

Previously, LPVs have been detected in four of our galaxies: IC 1613 (Menzies et al. 2015), NGC 147, NGC 185 (Lorenz et al. 2011), and Sag DIG (Whitelock et al. 2018). We have classified 19 of these previously detected sources as highconfidence LPVs. The pulsation periods measured in these works are comparable to those measured in this work (Table 5).

\section{Discussion}

Our new sample of LPVs allows us to study the pulsation properties of evolved stars in metal-poor environments and how they are affected by other observable parameters. These results along, with previous observations, suggest that dust production is unaffected by metallicity.

\subsection{Dust and Pulsation}

The [3.6]-[4.5] color has been shown to scale approximately with the dust content (Paper II; Riebel et al. 2015). Within our sample, both pulsation period and (especially) pulsation amplitude also correlate well with [3.6]-[4.5] color $^{16}$ (Figure 4). This correlation has been seen in more metal-rich samples in the galaxy, Magellanic Clouds, M33, and Sgr dSph (Whitelock et al. 2006; McQuinn et al. 2007; Javadi et al. 2011a; McDonald et al. 2014; Riebel et al. 2015). We see in Figure 5 that our amplitudes are unaffected by changes in

\footnotetext{
${ }^{16}$ Pulsation amplitude and period have also been seen to scale with mass-loss rate (Javadi et al. 2013; Goldman et al. 2017).
}

metallicity. Based on these relationships, it follows that dust production should also be unaffected by metallicity.

Uncertainties - There are uncertainties underlying our assumptions of dust production and metallicity. Given that most of our LPVs are expected to be carbon stars, we can only claim that dust production is unaffected by metallicity for carbon stars. The [3.6]-[4.5] color, a key metric in this analysis, will also depend on the dust temperature and wind speed, and the opacity of the dust may also differ at lower metallicities (McDonald et al. 2011, 2019). In determining the impact of metallicity on the dust production, we have assumed metallicities for our sample that were derived primarily from samples of RGB stars. These stars represent populations older and more metal-poor than our intermediate-mass LPVs. We expect the true metallicities of our LPVs to lie between these metallicities and ISM gas-phase oxygen abundances (shown in Table 1), yet neither show a correlation with amplitude.

\subsection{IR P-L Relation}

Wood (2015) reviewed what is known about the $P-L$ sequences of variable stars and suggested an evolutionary scenario with the current mass decreasing toward a longer period at a given luminosity. This allows us to follow the amount of mass that has been lost as a star moves toward the latest stages of its evolution.

Figure 6 shows period with respect to luminosity for our LPVs, with [3.6]-[4.5] color and metallicity in color. Our sample spans $\gtrsim 1$ dex in metallicity, providing a first look at how the IR $P-L$ relation behaves at very low metallicity. Compared to the SAGE+MACHO sample (Riebel et al. 2010), most of our LPVs follow the fundamental-mode sequence. We find that the reddest objects fall below the fundamental mode at $3.6 \mu \mathrm{m}$, a phenomenon that has also been seen in LPVs in the Magellanic Clouds (Ita et al. 2004b; Ita \& Matsunaga 2011) and IC 1613 (Whitelock et al. 2017). These sources, likely obscured by circumstellar extinction, have a decreased $3.6 \mu \mathrm{m}$ flux. In particular, IC 10 has a high number of reddened 


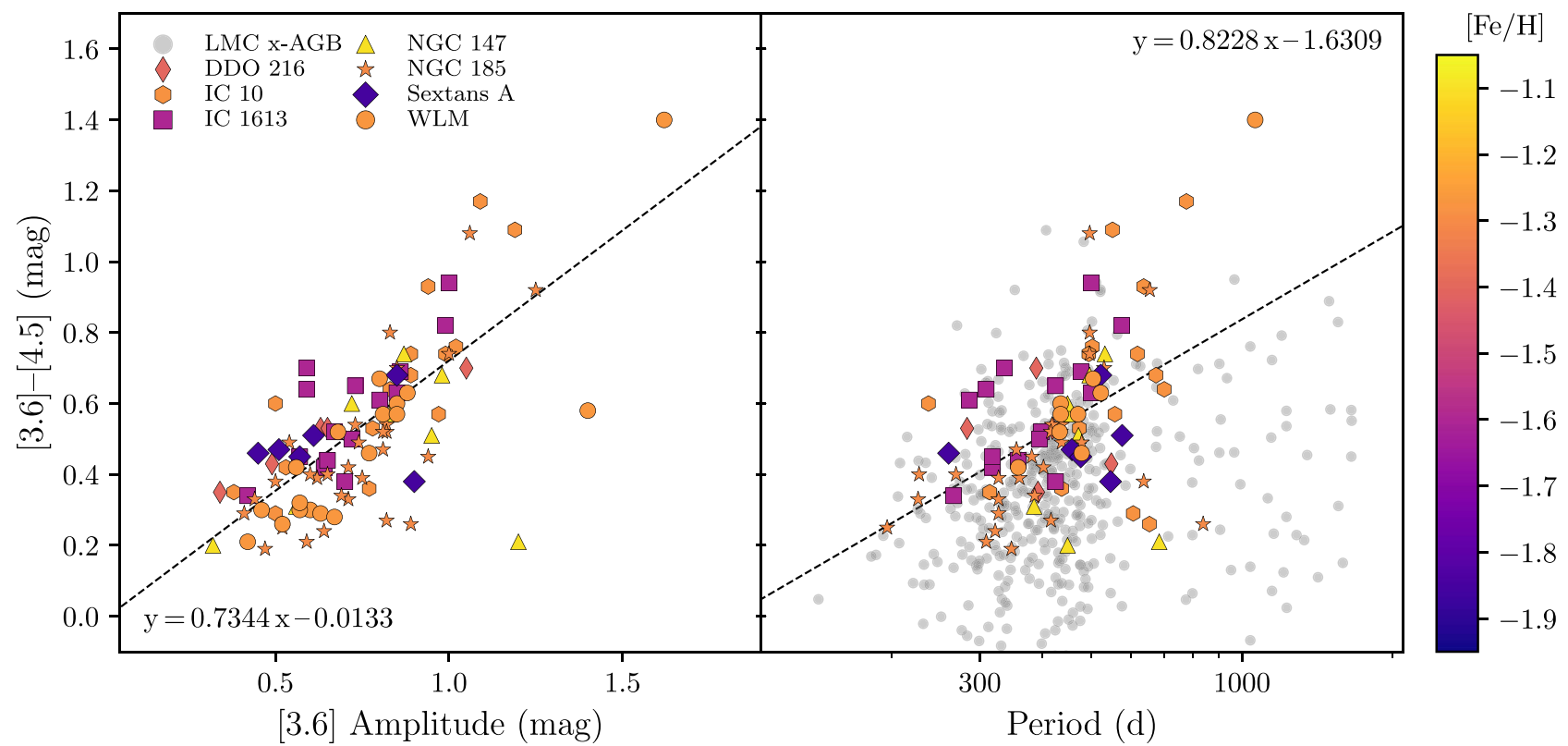

Figure 4. Pulsation amplitude and period of our RF sources vs. their Spitzer [3.6]-[4.5] color (indicative of dust content), with metallicity shown in color. The best-fit results and lines are shown in the figure. Also shown are pulsation periods for the x-AGB sample from the LMC (Riebel et al. 2010); $3.6 \mu \mathrm{m}$ amplitudes were not measured. The low-confidence variables are shown in Figure 11. The cadence and number of epochs in the SAGE-Var survey (Riebel et al. 2015) are insufficient for getting reliable amplitudes, which may contribute to the scatter for the LMC sample.

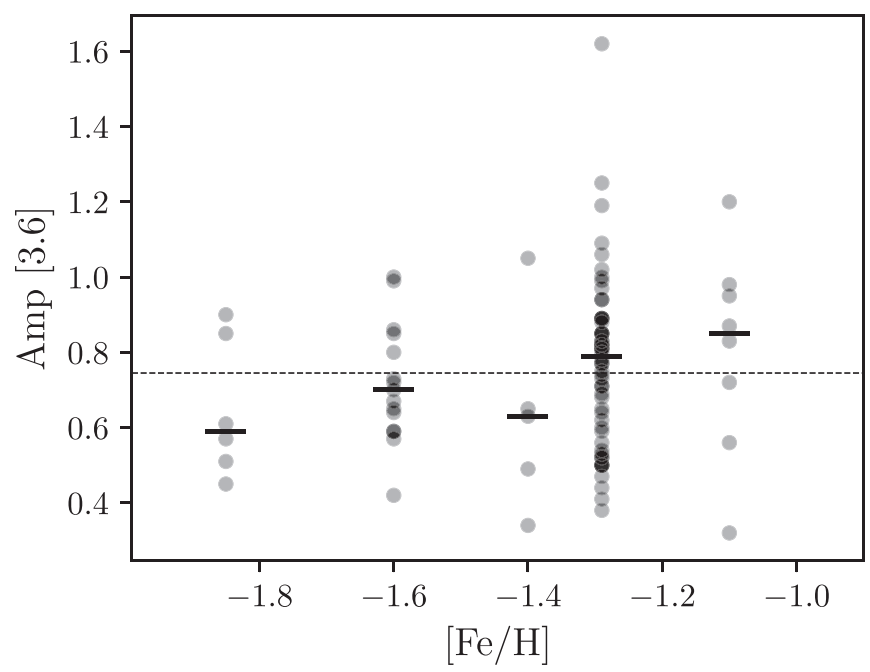

Figure 5. The $3.6 \mu \mathrm{m}$ best-fit amplitudes with respect to $[\mathrm{Fe} / \mathrm{H}]$. The dotted line shows the median amplitude of the reliable-fit sample. Black lines show the median amplitude in five metallicity bins. There is an apparent increase of the median amplitudes towards higher metallicity. However, this trend is dominated by stochastic sampling.

sources, which may be a combination of circumstellar and, to a lesser degree, interstellar extinction. It lies near the Galactic plane and has an estimated interstellar extinction of $A_{V} \sim 2.33 \mathrm{mag}$, which has been measured using a CMD analysis (Weisz et al. 2014). This should cause a mean approximate shift of $\sim 0.2 \mathrm{mag}$ in the IRAC magnitudes for which we have corrected for all sources in IC 10.

Our data suggest that, down to an $[\mathrm{Fe} / \mathrm{H}]=-1.85$, the $P-L$ relation of the fundamental mode (at $3.6 \mu \mathrm{m}$ ) is largely unaffected by metallicity. This suggests that the fundamental mode may be a robust tool for measuring distances to galaxies in the IR (e.g., Yuan et al. 2018). Since dusty AGB stars are among the brightest IR sources in galaxies, this technique can reach more distant galaxies than what can currently be measured with tip of the red giant branch (TRGB) distance estimates. However, there is an $\sim 1$ mag uncertainty in the absolute magnitude stemming from the width of the fundamental-mode sequence at $3.6 \mu \mathrm{m}$. The spread of the sequence may be a result of differences in the current mass as a result of mass loss. For the DUSTiNGS RF sources and x-AGB stars in the LMC that are firmly on the fundamental mode, we calculate the standard errors of the best fit of the x-AGB sample of 0.066 and 0.022 , respectively. This excludes several shorter-period and fainter LPVs and several strongly affected by the circumstellar dust. The smaller standard error we calculate for the LMC sample is expected, given the galaxy's larger and more complete sample and more accurately known interstellar extinction and distance. We modeled the LMC x-AGB $P-L$ relation with three parameters: slope, intercept, and intrinsic scatter. We used a first-order Student $t$ likelihood function, which has more weight in the tails than a Gaussian and is therefore less vulnerable to the effects of outliers (Galliano 2018). We sampled this likelihood function using PYMC3, a Monte Carlo Markov chain package for Python (Salvatier et al. 2016), with 5000 steps along 15 independent chains. Equation (1) shows the fit of the fundamental-mode $x$-AGB sample, calculated with a scatter of $0.25 \pm 0.01$; the fit is also shown in Figure 6:

$$
M_{3.6}=-5.26_{-0.14}^{+0.15} \log P+4.42_{-0.38}^{+0.38}
$$

Using the intercept error, this translates to a $4 \%$ uncertainty on the distance to a galaxy measured using the LMC $P-L$ relationship. This is smaller than the $\sim 8 \%$ uncertainty typically measured using the TRGB method (e.g., McQuinn et al. 2013) but uses only sources firmly on the fundamental mode. This may provide a more accurate tool at larger distances.

Using a similar approach as in Equation (1), we have also fitted the same LMC x-AGB sample at $4.5 \mu \mathrm{m}$ with a scatter of 


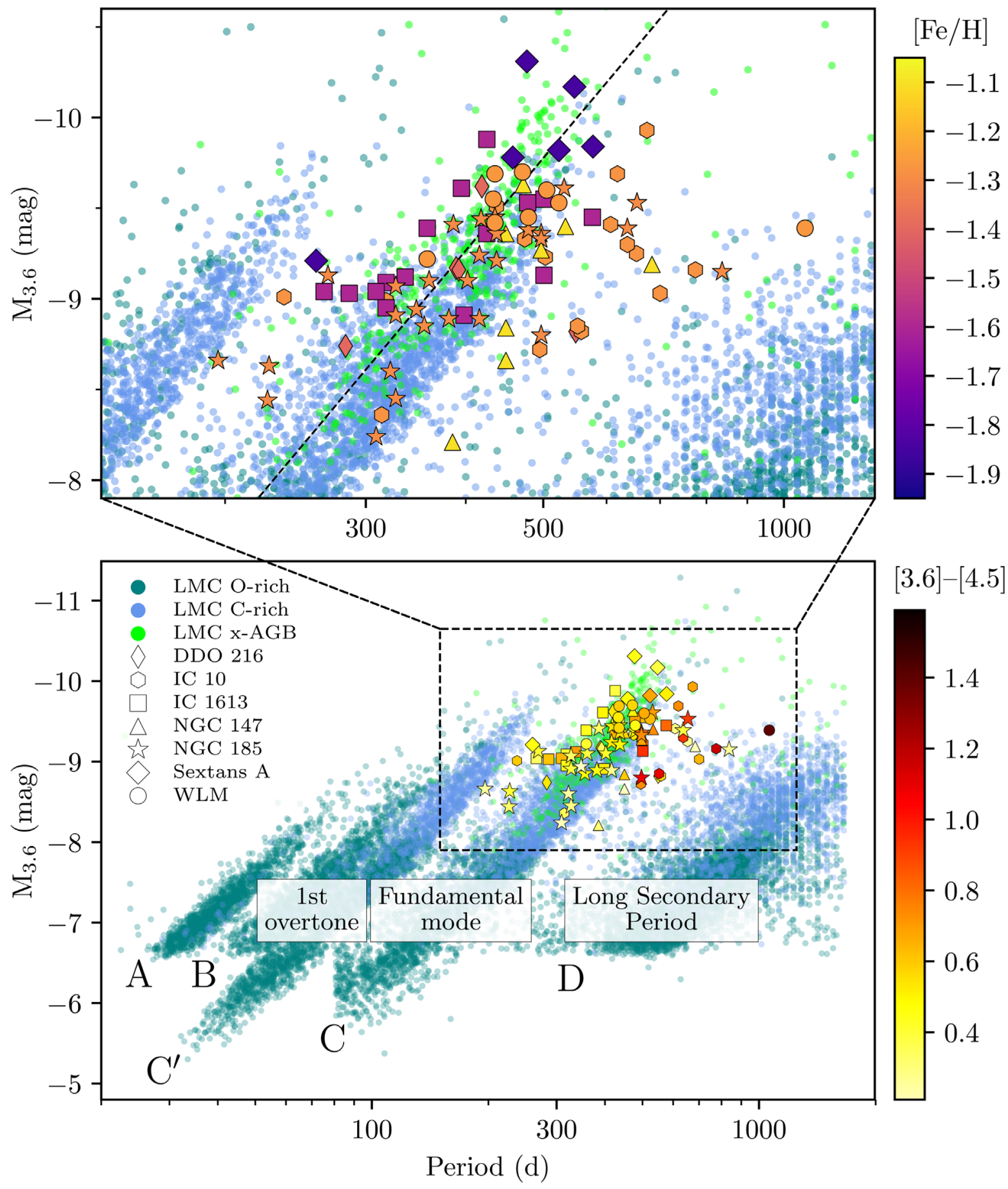

Figure 6. The $P-L$ relation of the high-confidence DUSTiNGS sample, with the color of the symbols showing the metallicity (top) and [3.6]-[4.5] color (bottom), as well as galaxy membership, indicated by shapes. We show the MACHO+SAGE sample from Riebel et al. (2010) containing oxygen- and carbon-rich AGB stars, as well as more evolved and dusty $\mathrm{x}$-AGB stars of both spectral types from the DUSTiNGS and MACHO+SAGE samples. Also shown is the best fit of the LMC x-AGB sources that are clearly fundamental-mode pulsators. Here $M_{3.6}$ was calculated using the midline value of the best-fit light curve for the DUSTiNGS sample.

$0.35 \pm 0.01:$

$$
M_{4.5}=-5.79_{-0.21}^{+0.20} \log P+5.44_{-0.53}^{+0.54} \text {. }
$$

At $4.5 \mu \mathrm{m}$ (Figure 7), the RF LPVs appear to be associated with a shifted fundamental-mode sequence significantly affected by circumstellar dust. This dust will veil molecular features like CO, known to affect the $P-L$ relation in Cepheids (Scowcroft et al. 2011; Blum et al. 2014). This shift off of the fundamental mode suggests that it may be more challenging to use this wavelength region for measuring distance using the brightest and dustiest stars.

\subsection{Classification of Stellar Chemistry with the HST}

Some of our RF LPV sources were previously observed with the Hubble Space Telescope (HST; Paper IV); Figure 15 in Appendix $\mathrm{C}$ shows the footprints of these observations. Medium-band optical photometry in the F127M, F139M, and 


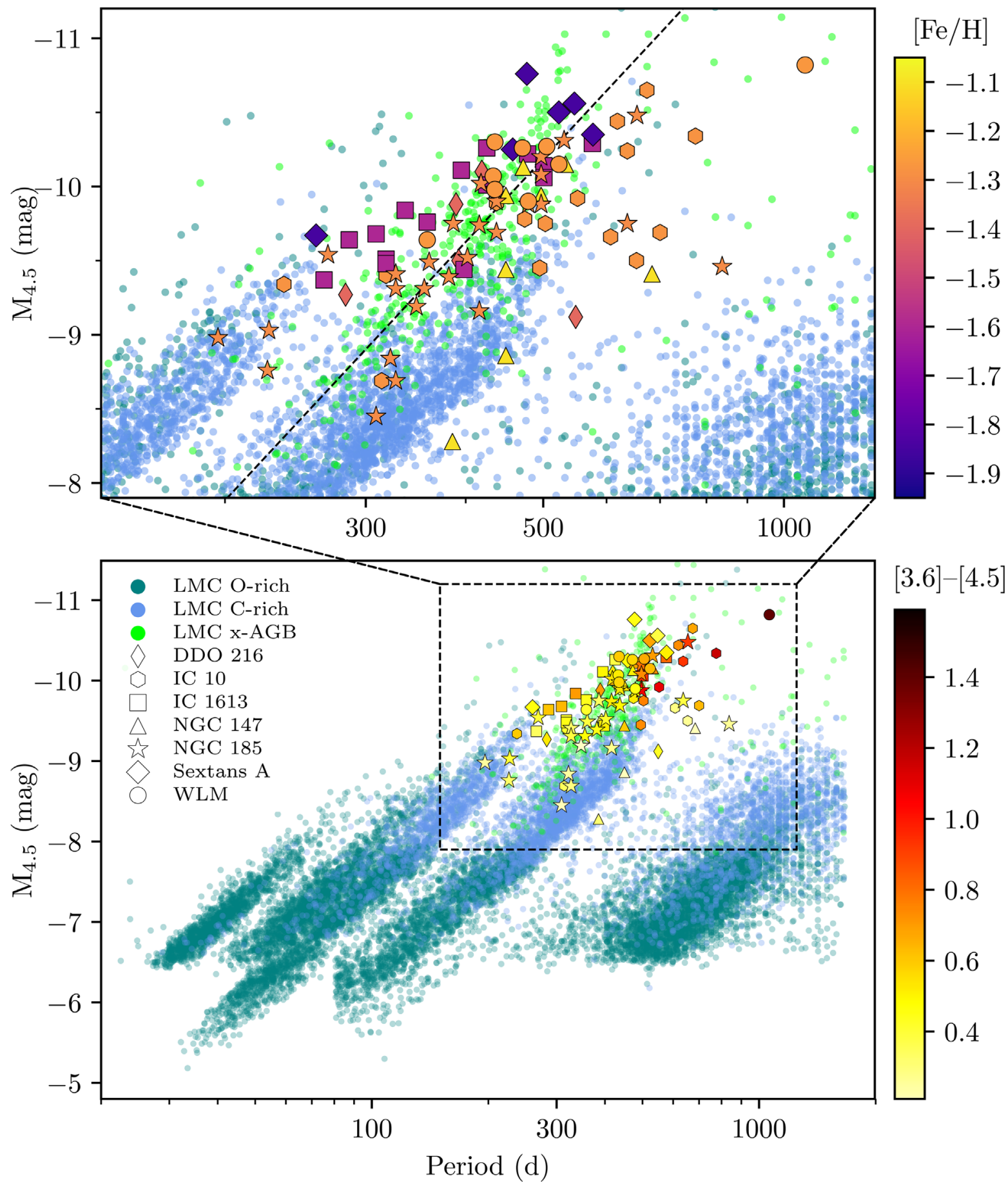

Figure 7. Same as Figure 6, but showing $4.5 \mu \mathrm{m}$ magnitudes. Dusty stars get brighter at $4.5 \mu \mathrm{m}$, creating a sequence more in line with the other sources. We see a tighter sequence than the sequence at $3.6 \mu \mathrm{m}$, which is affected by the [3.6]-[4.5] color.

F153M bands was used to categorize evolved stars by their chemical type (Paper IV). For our sample of RF LPVs, eight have $H S T$ counterparts with chemical types, all of which were found to be carbon-rich (Table 6). We found counterparts for nine sources with IEs, which include two oxygen-rich sources and seven carbon-rich sources with UFs. Theoretical models predict that most of these AGB populations will be dominated by carbon stars (Paper IV), with many fewer higher-mass oxygen-rich sources as a result of their metal-poor environments (Dell'Agli et al. 2016, 2018, 2019) and star formation histories (Hamedani Golshan et al. 2017; Javadi et al. 2017;
Goldman et al. 2018; Hashemi et al. 2019). Additionally, they may be too obscured in the near-IR, lack sufficient temporal coverage, or not covered in the HST observations. The fact that oxygen-rich LPVs have not been detected here is not proof that they do not exist.

\subsection{Individual Sources}

We have discovered several sources with particularly interesting light curves (Figure 8). And IX is the most metalpoor galaxy in our sample, and we detected one LPV candidate, And IX 5000004, that has a clear variability and is one of the 

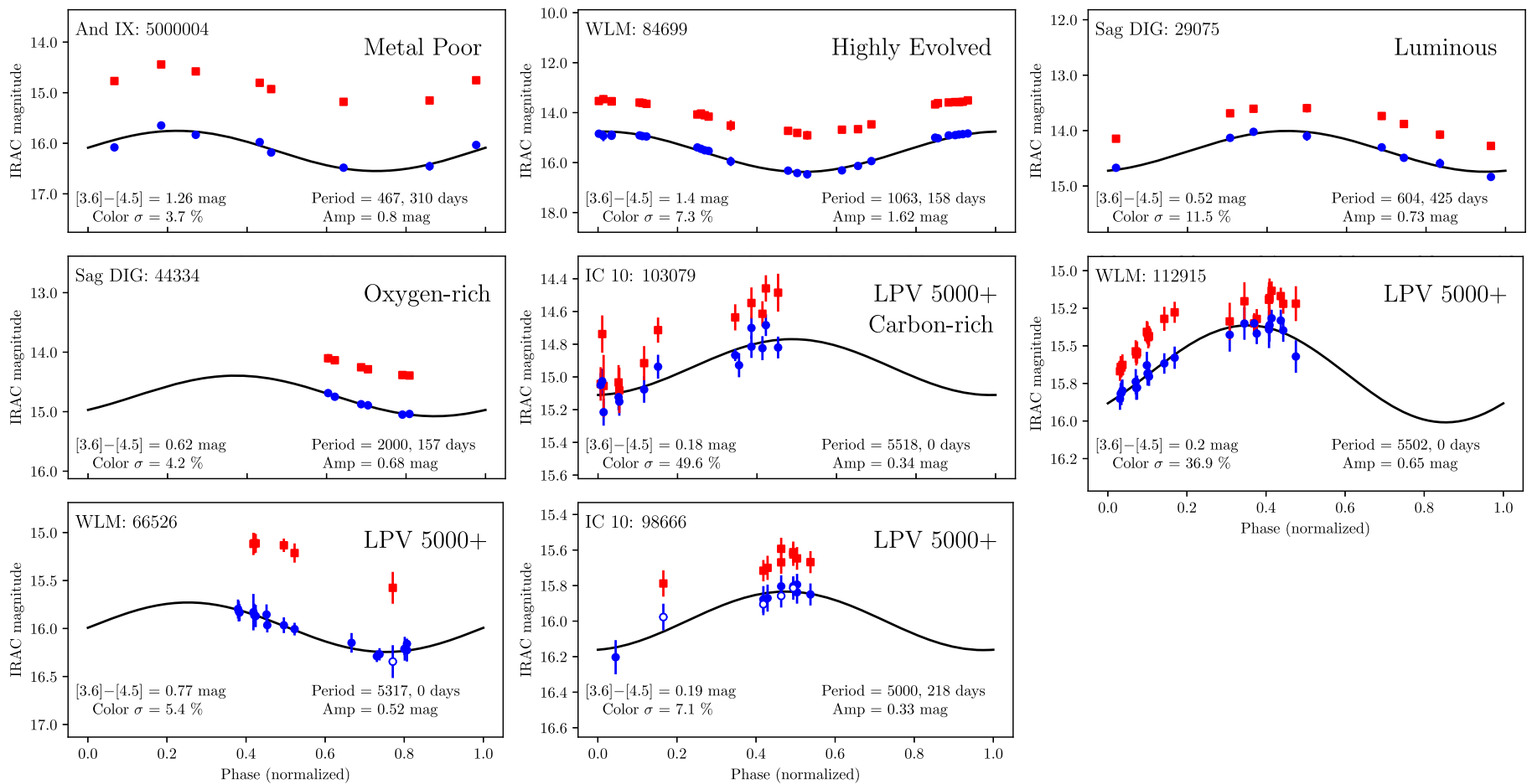

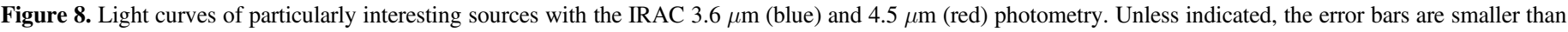

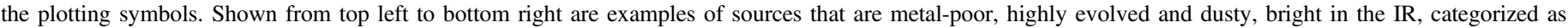
oxygen-rich, and the four LPV 5000+ sources. The LPV 5000+ source IC 10103079 also has a confirmed carbon-rich chemistry.

Table 6

DUSTiNGS Sources with Both Derived Pulsation Periods and Determined Chemical Type

\begin{tabular}{|c|c|c|c|c|c|c|c|c|c|c|c|c|}
\hline $\begin{array}{l}\text { (1) } \\
\text { Galaxy }\end{array}$ & $\begin{array}{l}\text { (2) } \\
\text { ID }\end{array}$ & $\begin{array}{r}(3) \\
\text { R.A. }\end{array}$ & $\begin{array}{c}\text { (4) } \\
\text { Decl. }\end{array}$ & $\begin{array}{l}\text { (5) } \\
\text { Type }\end{array}$ & $\begin{array}{c}(6) \\
\text { Period } \\
\text { (days) }\end{array}$ & $\begin{array}{c}\text { (7) } \\
{[3.6] \mathrm{amp}} \\
\text { (mag) }\end{array}$ & $\begin{array}{c}(8) \\
\text { F127M } \\
(\mathrm{mag})\end{array}$ & $\begin{array}{c}(9) \\
\text { F139M } \\
(\mathrm{mag})\end{array}$ & $\begin{array}{c}(10) \\
\text { F153M } \\
(\mathrm{mag})\end{array}$ & $\begin{array}{l}(11) \\
{[3.6]} \\
(\mathrm{mag})\end{array}$ & $\begin{array}{l}(12) \\
{[4.5]} \\
(\mathrm{mag})\end{array}$ & $\begin{array}{l}\text { (13) } \\
\text { Flag }\end{array}$ \\
\hline NGC 147 & 68407 & $00: 33: 25.61$ & $+48: 33: 15.1$ & $\mathrm{C}$ & 449 & 0.83 & 20.18 & 19.71 & 19.35 & 14.98 & 14.43 & $\mathrm{RF}$ \\
\hline NGC 147 & 112918 & 00:33:05.55 & $+48: 28: 37.2$ & $\mathrm{C}$ & 385 & 0.54 & 19.45 & 19.09 & 18.80 & 16.24 & 15.94 & $\mathrm{RF}$ \\
\hline DDO 216 & 96974 & $23: 28: 41.54$ & $+14: 44: 45.9$ & $\mathrm{C}$ & 392 & 0.34 & 19.76 & 19.24 & 18.94 & 15.70 & 15.35 & $\mathrm{RF}$ \\
\hline DDO 216 & 98916 & $23: 28: 40.97$ & $+14: 44: 01.3$ & $\mathrm{C}$ & 283 & 0.63 & 20.36 & 19.90 & 19.48 & 16.08 & 15.55 & $\mathrm{RF}$ \\
\hline DDO 216 & 101247 & $23: 28: 40.29$ & $+14: 44: 38.1$ & $\mathrm{C}$ & 419 & 0.65 & 19.26 & 18.80 & 18.33 & 15.29 & 14.76 & $\mathrm{RF}$ \\
\hline Sextans A & 86434 & 10:11:00.77 & $-04: 41: 54.0$ & C & 458 & 0.51 & 20.18 & 19.71 & 19.54 & 16.01 & 15.54 & $\mathrm{RF}$ \\
\hline Sextans A & 90941 & 10:10:59.20 & $-04: 42: 23.0$ & C & 260 & 0.45 & 20.27 & 19.82 & 19.67 & 16.53 & 16.07 & $\mathrm{RF}$ \\
\hline Sextans A & 98908 & $10: 10: 56.45$ & $-04: 41: 33.0$ & C & 477 & 0.57 & 20.27 & 19.79 & 19.41 & 15.57 & 15.12 & $\mathrm{RF}$ \\
\hline IC 10 & 101088 & 00:20:15.71 & $+59: 16: 00.9$ & $\mathrm{C}$ & $215^{\mathrm{a}}$ & 0.56 & 19.99 & 19.47 & 19.19 & 15.70 & 15.33 & IE \\
\hline IC 10 & 101812 & $00: 20: 15.24$ & $+59: 15: 59.3$ & $\mathrm{C}$ & $210^{\mathrm{a}}$ & 0.41 & 18.90 & 18.33 & 18.08 & 14.89 & 14.68 & IE \\
\hline IC 10 & 109003 & $00: 20: 10.26$ & $+59: 20: 10.4$ & $\mathrm{C}$ & $170^{\mathrm{a}}$ & 0.32 & 18.07 & 17.65 & 17.46 & 14.65 & 14.27 & IE \\
\hline IC 10 & 109003 & $00: 20: 10.17$ & $+59: 20: 11.6$ & M & $170^{\mathrm{a}}$ & 0.32 & 19.54 & 19.24 & 18.80 & 14.65 & 14.27 & IE \\
\hline IC 10 & 111369 & 00:20:08.84 & $+59: 16: 57.6$ & $\mathrm{C}$ & $170^{\mathrm{a}}$ & 0.69 & 19.22 & 18.70 & 18.47 & 15.58 & 15.03 & IE \\
\hline IC 10 & 117441 & 00:20:04.56 & $+59: 21: 11.8$ & $\mathrm{C}$ & $132^{\mathrm{a}}$ & 0.44 & 18.54 & 18.15 & 17.98 & 15.92 & 15.74 & IE \\
\hline NGC 147 & 55735 & $00: 33: 32.28$ & $+48: 32: 47.3$ & $\mathrm{C}$ & $133^{\mathrm{a}}$ & 0.31 & 18.69 & 18.34 & 18.20 & 15.92 & 15.70 & IE \\
\hline NGC 147 & 113288 & 00:33:05.40 & $+48: 30: 18.1$ & $\mathrm{C}$ & $317^{\mathrm{a}}$ & 0.49 & 18.08 & 17.69 & 17.55 & 15.83 & 15.63 & IE \\
\hline Sag DIG & 44334 & 19:29:57.95 & $-17: 40: 17.0$ & M & $2000^{\mathrm{a}}$ & 0.68 & 18.03 & 17.72 & 17.26 & 14.88 & 14.26 & IE \\
\hline IC 10 & 65446 & $00: 20: 39.81$ & $+59: 16: 39.2$ & $\mathrm{C}$ & $854^{\mathrm{a}}$ & 0.35 & 19.52 & 19.04 & 18.73 & 15.79 & 15.53 & UF \\
\hline IC 10 & 65548 & $00: 20: 39.74$ & $+59: 17: 25.3$ & $\mathrm{C}$ & $136^{\mathrm{a}}$ & 0.43 & 19.84 & 19.37 & 19.03 & 15.73 & 15.33 & UF \\
\hline IC 10 & 73607 & $00: 20: 34.08$ & $+59: 15: 58.1$ & $\mathrm{C}$ & $695^{\mathrm{a}}$ & 0.41 & 19.40 & 18.89 & 18.53 & 15.32 & 15.10 & UF \\
\hline IC 10 & 107394 & $00: 20: 11.34$ & $+59: 21: 14.5$ & $\mathrm{C}$ & $555^{\mathrm{a}}$ & 0.80 & 20.14 & 19.64 & 19.39 & 16.01 & 15.58 & UF \\
\hline IC 10 & 114178 & 00:20:06.78 & $+59: 19: 57.2$ & $\mathrm{C}$ & $378^{\mathrm{a}}$ & 0.40 & 19.46 & 18.99 & 18.66 & 15.70 & 15.37 & UF \\
\hline Sextans A & 91449 & $10: 10: 59.05$ & $-04: 40: 14.0$ & $\mathrm{C}$ & $524^{\mathrm{a}}$ & 0.36 & 20.61 & 20.07 & 19.77 & 16.07 & 15.47 & UF \\
\hline IC 10 & 103079 & $00: 20: 14.25$ & $+59: 19: 07.0$ & $\mathrm{C}$ & $\ldots$ & $\ldots$ & 18.93 & 18.41 & 18.20 & 15.14 & 14.98 & LPV $5000+$ \\
\hline
\end{tabular}

Note. The DUSTiNGS LPVs with HST photometry used to determine the spectral type (Paper IV). Columns 11 and 12 show the average magnitudes of all of the 3.6 and $4.5 \mu \mathrm{m}$ epochs. Flag is the same as in Table 3.

${ }^{a}$ Value unlikely due to the low confidence of the fit solution. 
reddest sources in our sample. It lies near the outer regions of M31's disk at $37 \mathrm{kpc}$ from the galaxy's center and, while unlikely, could be a metal-rich M31 interloper. More epochs are needed to constrain the precise period, and spectroscopy is needed to confirm its membership in And IX.

Our most massive source is WLM 84699, with a pulsation period of 1063 days, fitted peak-to-peak pulsation amplitude of $1.62 \mathrm{mag}$ at $3.6 \mu \mathrm{m}$, and [3.6]-[4.5] color of $1.6 \mathrm{mag}$. This is indicative of a very late stage of evolution, a high mass-loss rate, and a high dust production rate. This source was also analyzed in Karambelkar et al. (2019) and lies between the lower-mass population of Mira variables and what they claim are massive AGB stars in the $P-L$ diagram. These massive AGB stars are also in galaxies with younger populations than WLM, making it a particularly interesting target for spectroscopic follow-up and an analysis of the source's dust composition.

While not as evolved, Sag DIG 29075 is our most luminous source and also quite metal-poor, but more observations are needed to constrain the period. Another source within Sag DIG, 44334, was previously categorized in Paper IV as oxygen-rich, making it one of the most metal-poor and dusty oxygen-rich evolved stars known. This source has a known pulsation period of 950 days from ground-based observations in the near-IR (Whitelock et al. 2018). We lack enough IR data to further constrain the period. However, the clear variable nature of the source, together with its red color, strongly suggest that it is producing dust.

The remaining four sources in Figure 8 have been categorized as LPV 5000+ and show a gradual change over the entirety of the light curve. While these sources may be shorter-period evolved stars with a coincidental cadence, they may also have dominant pulsation periods that are considerably longer than a typical AGB star $(P \gtrsim 2000$ days $)$ or may just be growing or diminishing in brightness with no periodicity. Only one of the LPV 5000+ sources (IC 10 103079) has been confirmed as carbon-rich and thus an AGB star (Paper IV).

\subsection{Long Secondary Period}

Distinct from the LPV 5000+ are sources with LSPs. The sequence hosts less-evolved stars, as well as TP-AGB stars, with periods between 400 and 1200 days. Recent work by Wood (2015) and Trabucchi et al. (2017, 2018) has improved our understanding of how stars evolve along these sequences, but the mechanism that drives the LSP is still unclear. It is now known that stars with primary periods associated with the LSP, sequence $\mathrm{D}$, have secondary periods that lie in the middle of the first-overtone sequence made up of $\mathrm{B}$ and $\mathrm{C}^{\prime}$. The reason for the appearance of the sequence-D period in these stars is unknown but may arise from convection, binarity, or changes in the internal chemistry of the star (Nicholls et al. 2009; Mathias et al. 2018). The pulsation behavior of our LPV 5000+ sources may be that of an LSP.

\subsection{High-redshift Dust}

Our observations provide further evidence of the evolved nature of the dusty sources found in metal-poor galaxies and for significant AGB dust production in these environments. The lowest metallicities of our LPV sample are characteristic of galaxies $\sim 12.3 \mathrm{Gyr}$ ago and redshifts of $z \sim 5$ (Rafelski et al. 2012; Poudel et al. 2017). Paper IV identified both carbon- and oxygen-rich evolved stars at low metallicity. As luminous oxygen-rich sources are more massive than their lower-mass carbon-rich counterparts, they are capable of injecting dust into the ISM as early as $30 \mathrm{Myr}$ after forming (for a $10 M_{\odot}$ star), while carbon stars are expected to take longer (as soon as 200-300 Myr; Sloan et al. 2009). Most dust evolution models ignore dust produced by metal-poor oxygen-rich stars.

While we expect that AGB stars may produce dust in this regime, it is unclear whether they are the dominant dust producers. Supernovae may produce considerable dust; however, their net contribution is still unclear due to the unknown efficiency of dust destruction (Lakićević et al. 2015; Temim et al. 2015). Another alternative is dust produced by grain growth within the ISM (Zhukovska et al. 2008). However, a theory as to how the grains grow and what seeds their nucleation has yet to be identified. While the pulsation properties and $3-5 \mu \mathrm{m}$ observations highlight the important role that AGB stars play in dust production, longer-wavelength observations are critical to constraining the amount of cooler dust surrounding these stars. More observations are needed to confirm that AGB stars are capable of producing dust out to $z \sim 6$. The soon-to-launch James Webb Space Telescope (JWST) will allow us to study Local Group AGB samples in much greater detail. In particular, observations with the JWST Mid-infrared Instrument (Rieke et al. 2015) will be able to obtain photometry for every star in this sample out to $25 \mu \mathrm{m}$ (Jones et al. 2017).

\section{Conclusion}

This survey has provided the first IR light curves of dusty evolved stars in metal-poor environments. We surveyed 10 metal-poor dwarf galaxies within $1.5 \mathrm{Mpc}$ at 3.6 and $4.5 \mu \mathrm{m}$. By combining our multi-epoch observations with archival observations, we identified the dustiest evolved AGB stars within these galaxies, sources that may have been missed in the near-IR or optical surveys due to dust obscuration. We have identified 88 sources in seven of these galaxies as highconfidence LPV candidates, eight of which have been confirmed as carbon-rich.

We find that metallicity does not seem to have a strong impact on AGB pulsation or dust production. This has implications for the dust seen at high redshift and the origin of dust in the early universe. We also find that the fundamental mode of the IR $P-L$ relationship seems unaffected by metallicity, at least between one-half and one-hundredth solar. This suggests that the $P-L$ relation can be a useful tool in measuring distance. With IR observations with JWST, the $P-L$ relation can be used to confirm distances to Type Ia supernovae in distant galaxies, providing additional constraints on the Hubble constant $\left(H_{0}\right)$.

We would like to thank the SPIRITS team (PI: Mansi Kasliwal) for assistance with the inclusion of the SPIRITS data in four of our galaxies and Chris Clark for his help with the $P-L$ distance uncertainty calculation. This work is supported by Spitzer via grant GO11041 and the NASA Astrophysics Data Analysis Program grant No. NNX16AT56G. R.D.G. was supported by NASA and the United States Air Force. O.C.J. has received funding from the European Union's Horizon 2020 research and innovation program under Marie SklodowskaCurie grant agreement No. 665593 awarded to the Science and Technology Facilities Council. 

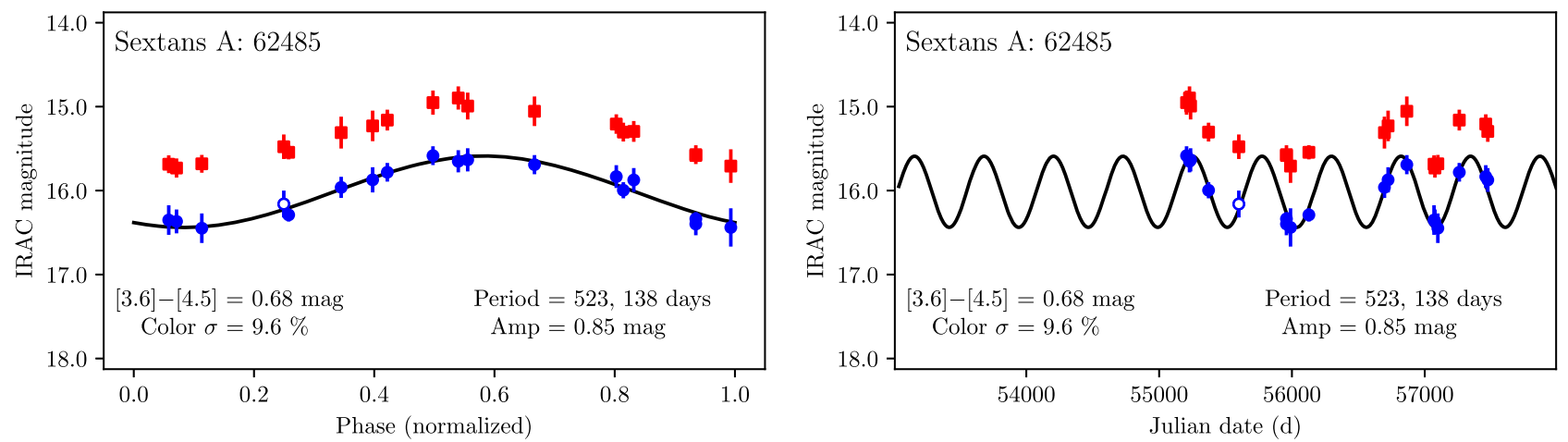

Figure 9. Our sample of high-confidence fit light curves. Shown are the IRAC $3.6 \mu \mathrm{m}$ (blue) and $4.5 \mu \mathrm{m}$ (red) photometry and the best-fitting periodogram fitted to the [3.6] data. Unless otherwise indicated, the data error bars are smaller than the plotting symbols. Also shown is our "simulated" photometry, denoted using open circles (see Section 3.2). The two numbers listed as period are the best- and second-best-fit values. Also shown is the best-fit $3.6 \mu \mathrm{m}$ amplitude. Sextans A (62485) is given as an example, with the phased light curve on the left and the unphased light curve on the right. The complete figure set (88 images) is available in the online journal.

(The complete figure set ( 88 images) is available.)

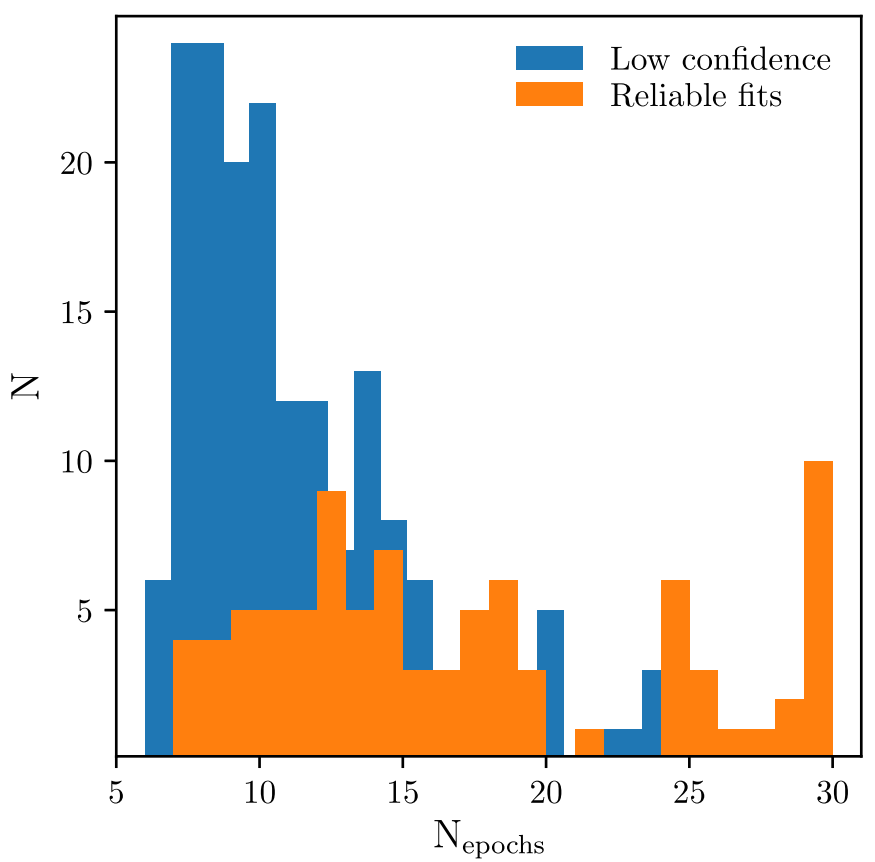

Figure 10. Histogram of the number of epochs for the RF and low-confidence LPVs. Light curves with less than 10 epochs are typically categorized as lowconfidence LPVs.

\section{Appendix A IR Light Curves}

This appendix shows the phased and unphased light curves of the RF LPVs (Figure 9). The IRAC $3.6 \mu \mathrm{m}$ (blue circles) light curves were fit with simple single-term sinusoidal functions using the Lomb-Scargle algorithm. Also shown are the corresponding $4.5 \mu \mathrm{m}$ magnitudes (red squares). Simulated $3.6 \mu \mathrm{m}$ photometry (see Section 3.2) are shown as open circles. Figure 10 shows the number of epochs for our RF and lowconfidence LPVs.

\section{Appendix B \\ Low-confidence DUSTiNGS Variables}

From our visual examination of our sources, we have categorized them into two groups: high- and low-confidence LPVs (described in 3.3). The low-confidence variables are those with IEs to constrain the light curve or a poor fit of the model to the data. For the low-confidence variables, we show the $P-L$ relation and how the pulsation behavior is affected by the [3.6]-[4.5] color in Figures 11, 13, and 14. Examples of low-confidence light curves are shown in Figure 12; high-confidence variables are shown in Figure 6. 


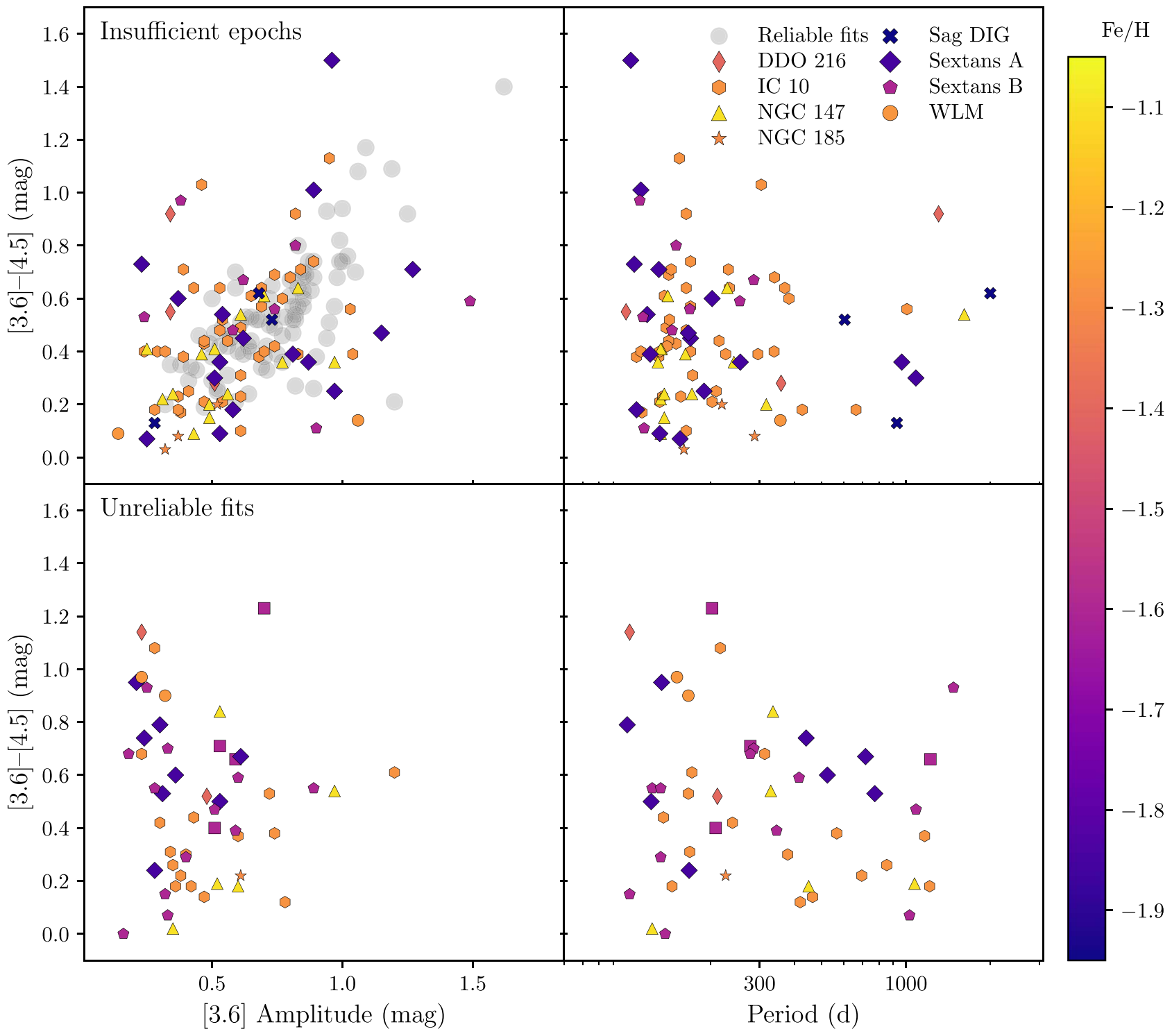

Figure 11. Same as Figure 4, but showing the sources designated as IE (top) and UF (bottom). While accurate periods could not be measured, the amplitudes are expected to be more accurate.
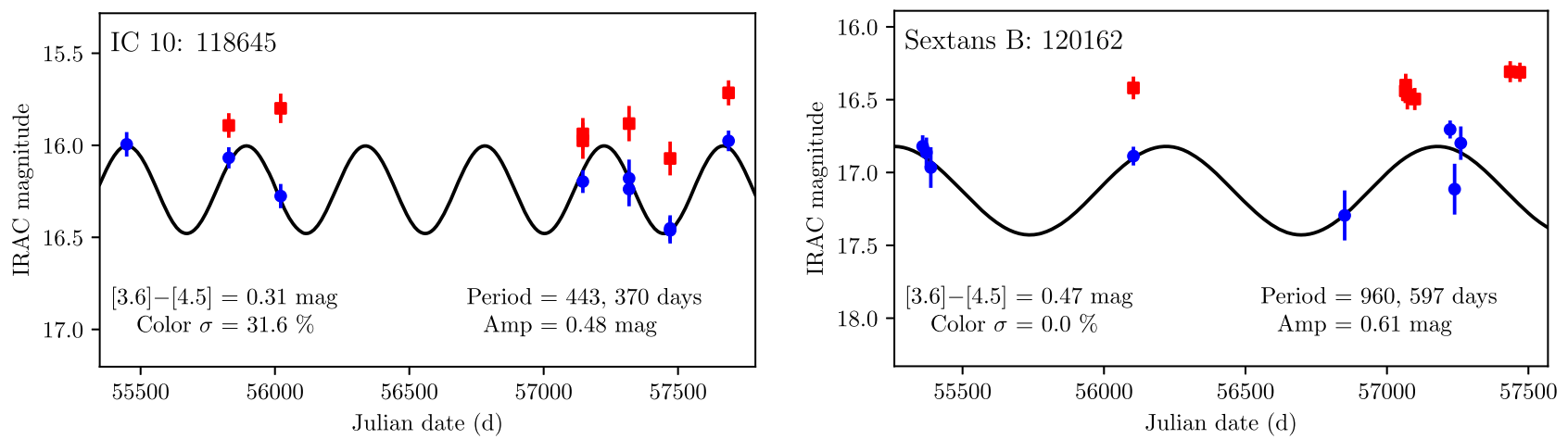

Figure 12. Examples of light curves with IEs (left) and a UF (right). The classification of IEs is only made if an RF source could plausibly be fit with a shorter or longer period. 


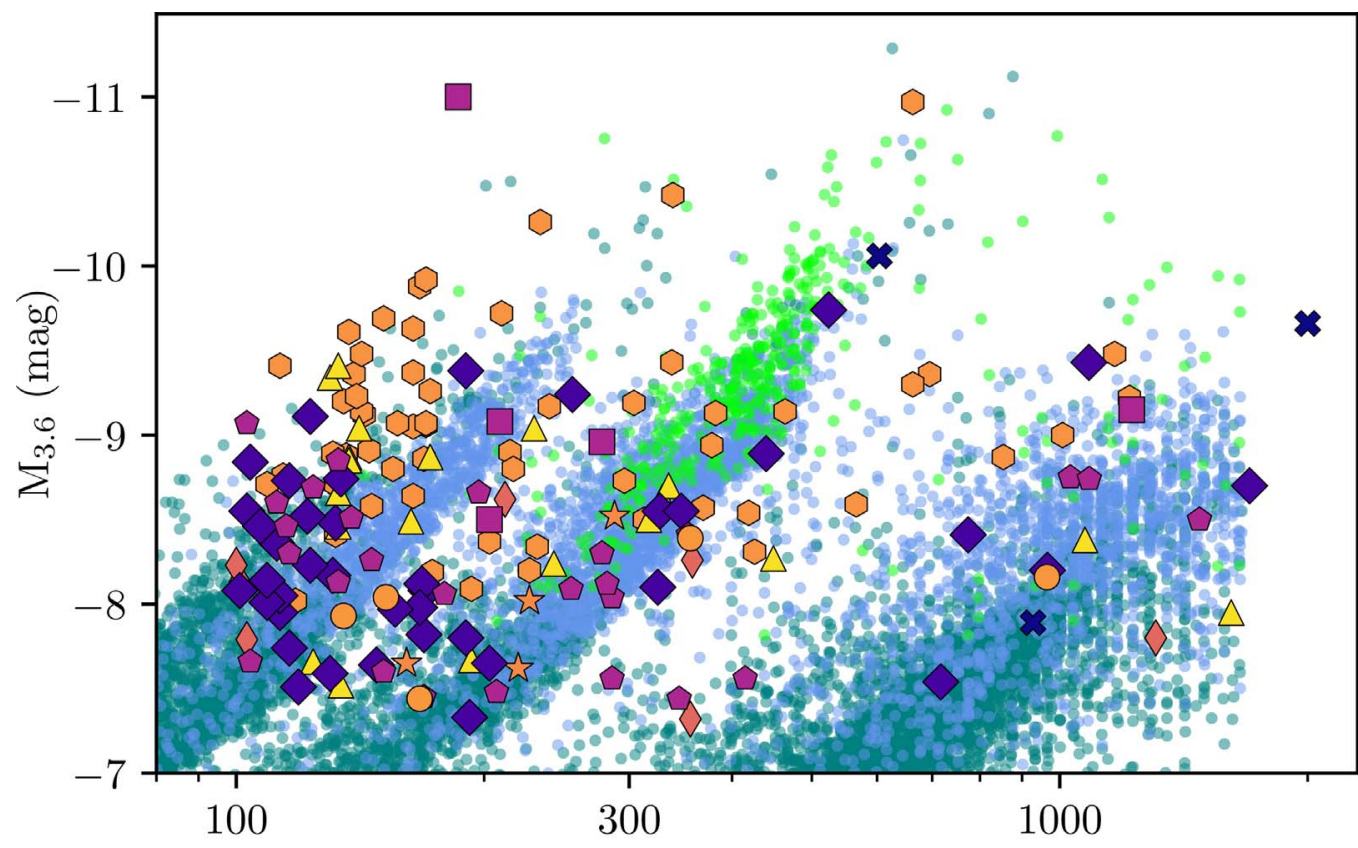

$[\mathrm{Fe} / \mathrm{H}]$
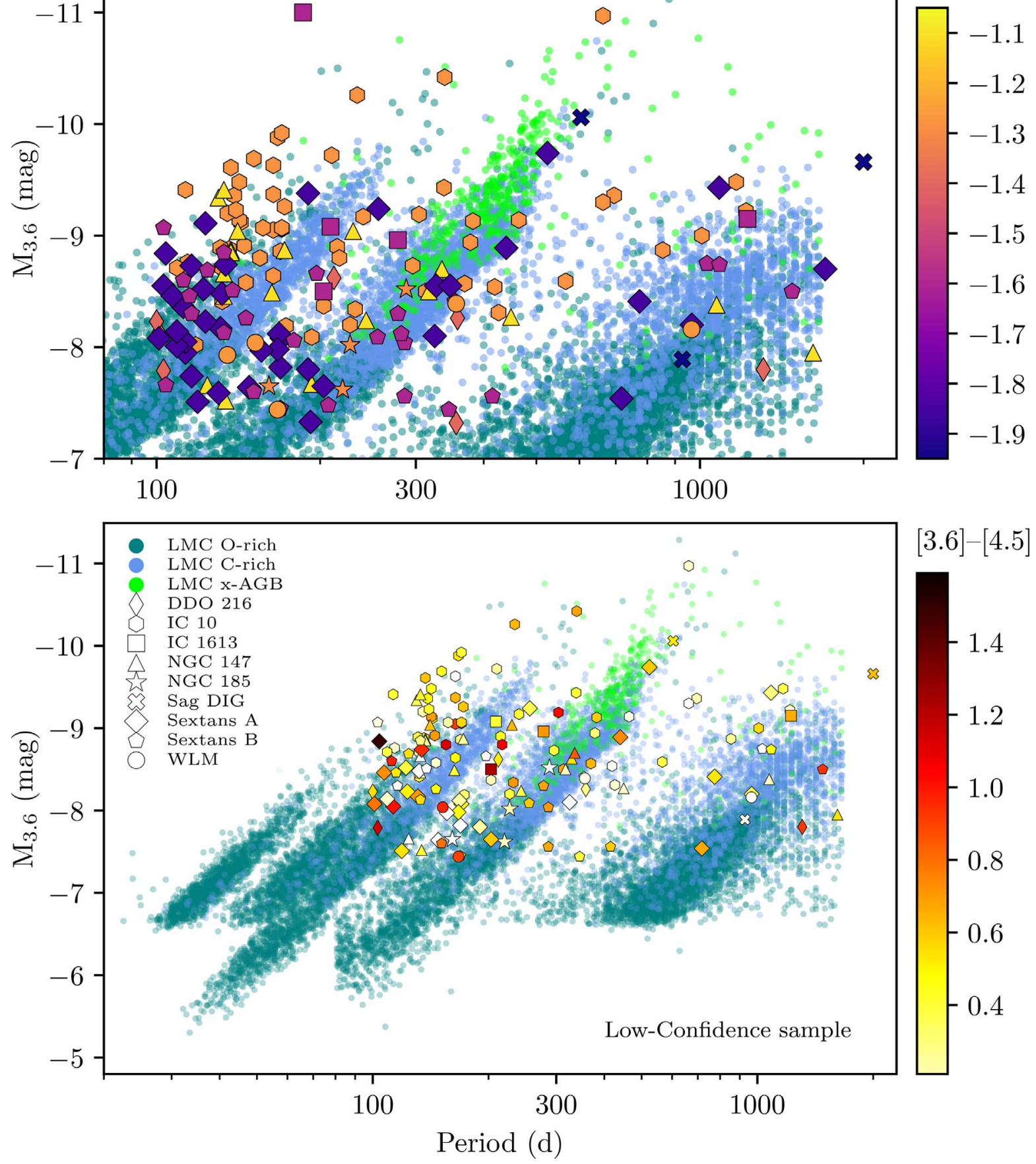

$[3.6]-[4.5]$

Figure 13. Same as Figure 6, but showing the low-confidence variables. 

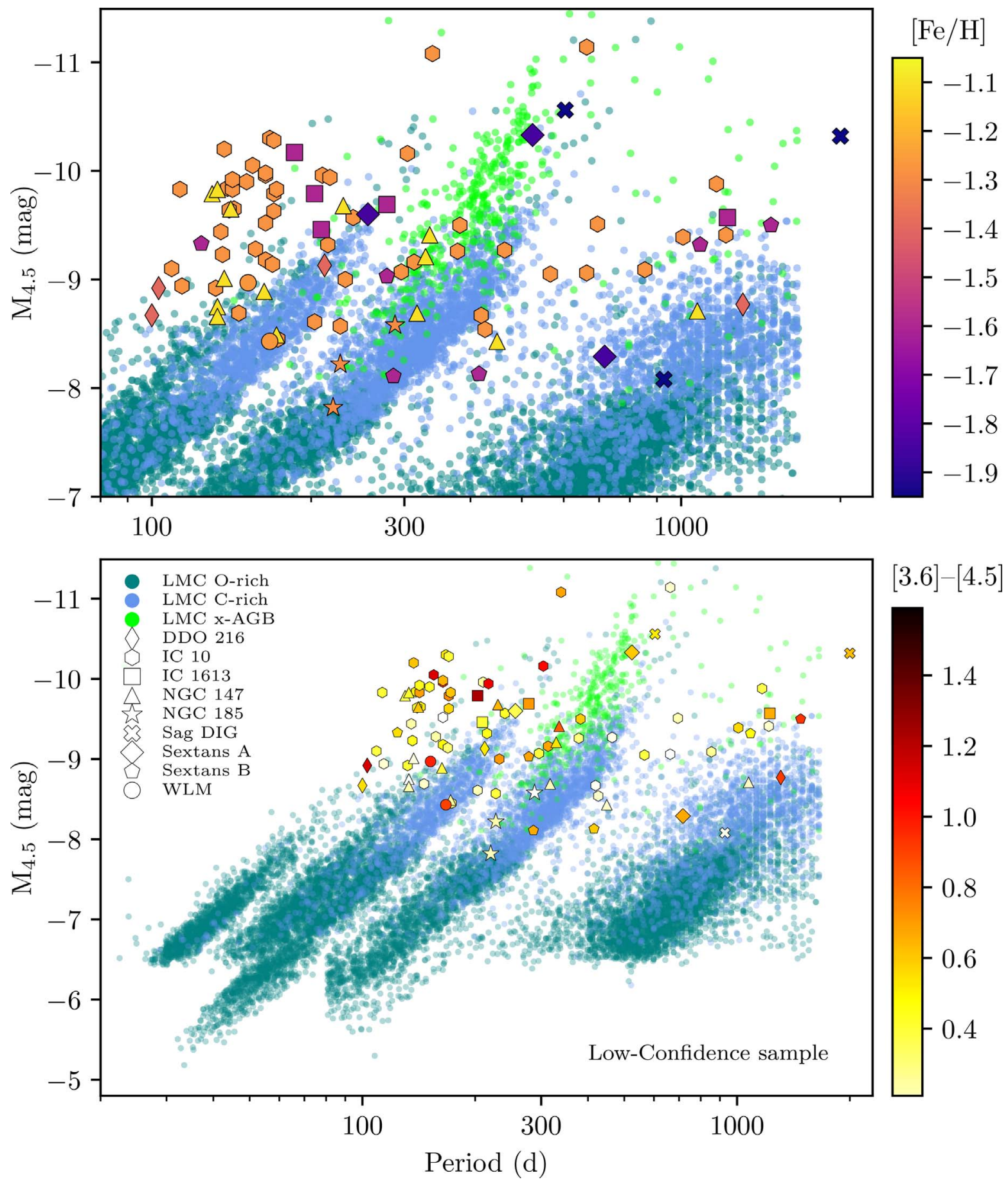

Figure 14. Same as Figure 13, but showing the $4.5 \mu \mathrm{m}$ magnitudes.

\section{Appendix C \\ DUSTiNGS Spatial Distribution}

We have mapped the spatial distribution of the high- and low-confidence DUSTiNGS LPVs on the Spitzer mosaics from Paper I (Figure 15). Also shown are the footprints of the HST observations used in Paper IV to disentangle the oxygen- and carbon-rich evolved AGB stars and the intersecting region that covers all six of the Cycle 11 observations. We have compared the LPVs detected in these intersection regions to the results of Paper II (Table 7) to understand how additional epochs have identified high-confidence variables. Paper II identified $2 \sigma$ and $3 \sigma$ variables using two epochs of data. This survey confirmed a subset of those variables (Table 7). Varying spatial coverage between epochs prevented all of the Paper II variables from being confirmed. However, the increase in the number of epochs in some regions resulted in the discovery of new variables (from 2 to 46 per galaxy) that were not identified in Paper II. Given our spatially and temporally uneven coverage, the variable stars we confirm here are likely a small subset of the total variable population in each galaxy. 

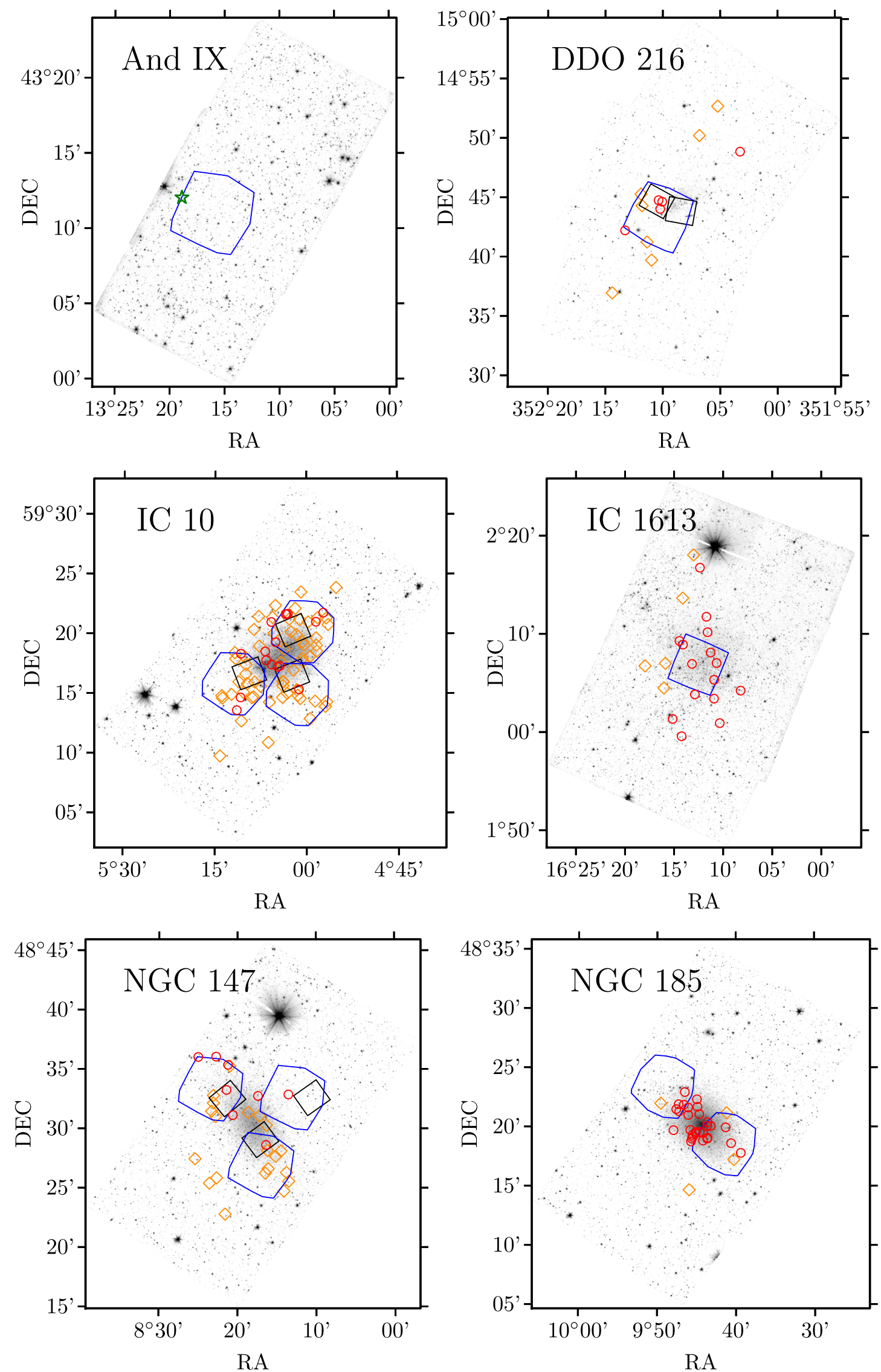

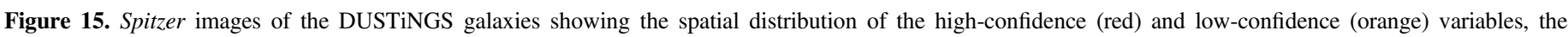

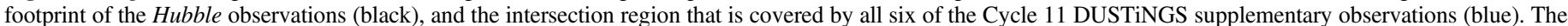
source And IX 46835 is shown with a green star. 

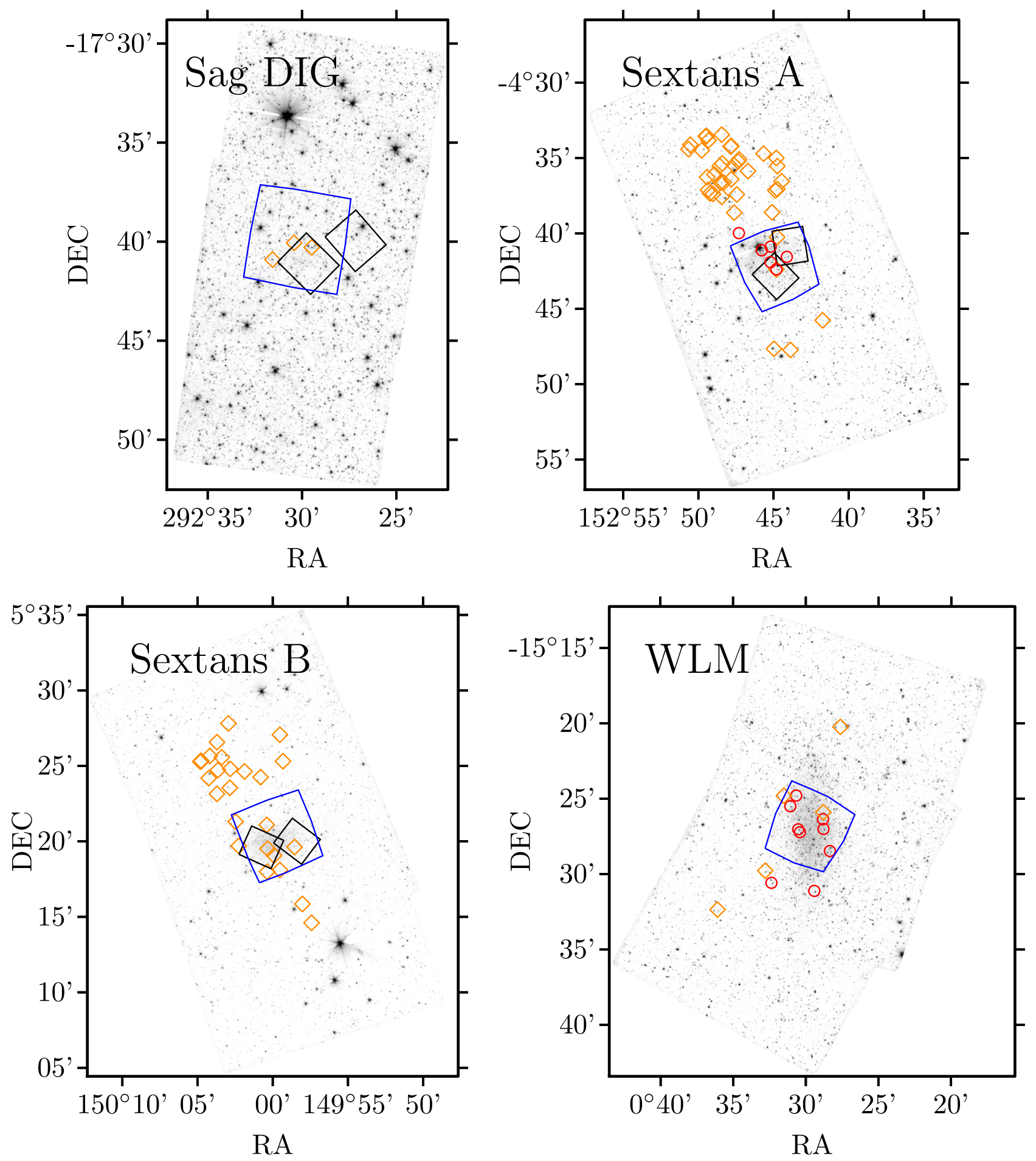

Figure 15. (Continued.) 
Table 7

DUSTiNGS Results That Were Covered in Every Epoch of the Cycle 11 DUSTiNGS Supplementary Data

\begin{tabular}{|c|c|c|c|c|c|c|c|}
\hline Galaxy & $\mathrm{RF}$ & IEs & UF & LPV $5000+$ & $\begin{array}{c}\text { Paper II } \\
2 \sigma \text { X-AGBs }\end{array}$ & $\begin{array}{c}\text { Paper II } \\
3 \sigma \text { X-AGBs }\end{array}$ & $\begin{array}{c}3 \sigma \text { x-AGBS Detected } \\
\text { in This Work }\end{array}$ \\
\hline And IX & 0 & 0 & 0 & 0 & 0 & 2 & $\ldots$ \\
\hline DDO 216 & 3 & 1 & 2 & 0 & 0 & 5 & $60 \%$ \\
\hline IC 1613 & 5 & 0 & 0 & 0 & 1 & 10 & $50 \%$ \\
\hline NGC 147 & 5 & 10 & 1 & 0 & 2 & 36 & $13 \%$ \\
\hline NGC 185 & 16 & 2 & 0 & 0 & 2 & 28 & $57 \%$ \\
\hline Sextans B & 0 & 3 & 4 & 0 & 2 & 19 & $\ldots$ \\
\hline WLM & 7 & 0 & 1 & 1 & 1 & 18 & $38 \%$ \\
\hline Total & 51 & $\ldots$ & $\ldots$ & $\ldots$ & $\ldots$ & 266 & $19 \%$ \\
\hline
\end{tabular}

Note. Here we list a subset of the results of the light-curve fitting that lie in the regions covered by all epochs in the Cycle 11 DUSTiNGS supplementary data (shown in blue in Figure 15). Also shown are the Paper II x-AGB stars that were also found in those regions and the percentage of those that were confirmed in this work.

The LPVs detected outside of these regions have sporadic temporal coverage and poorly constrained light curves, with the exception of a few sources with additional archival data. There is also a high number of low-confidence variables in Sextans A and Sextans B above the intersection region. This is due to a higher number of epochs covering these regions as opposed to in the south.

\section{ORCID iDs}

S. R. Goldman @ https://orcid.org/0000-0002-8937-3844

M. L. Boyer (1) https://orcid.org/0000-0003-4850-9589

K. B. W. McQuinn (1) https://orcid.org/0000-0001-5538-2614

J. Th. van Loon (ib https://orcid.org/0000-0002-1272-3017

E. D. Skillman (1) https://orcid.org/0000-0003-0605-8732

R. D. Gehrz (i) https://orcid.org/0000-0003-1319-4089

O. C. Jones (ib https://orcid.org/0000-0003-4870-5547

\section{References}

Alcock, C., Allsman, R. A., Alves, D., et al. 1997, ApJ, 486, 697 Bellazzini, M., Beccari, G., Fraternali, F., et al. 2014, A\&A, 566, A44 Bladh, S., Höfner, S., Aringer, B., \& Eriksson, K. 2015, A\&A, 575, A105 Blum, R. D., Mould, J. R., Olsen, K. A., et al. 2006, AJ, 132, 2034 Blum, R. D., Srinivasan, S., Kemper, F., Ling, B., \& Volk, K. 2014, AJ, 148, 86

Boyer, M. L., McQuinn, K. B. W., Barmby, P., et al. 2015a, ApJ, 800, 51 Boyer, M. L., McQuinn, K. B. W., Barmby, P., et al. 2015b, ApJS, 216, 10 Boyer, M. L., McQuinn, K. B. W., Groenewegen, M. A. T., et al. 2017, ApJ, 851,152

Clement, C. M., Muzzin, A., Dufton, Q., et al. 2001, AJ, 122, 2587

Dell'Agli, F., Di Criscienzo, M., Boyer, M. L., \& García-Hernández, D. A. 2016, MNRAS, 460, 4230

Dell'Agli, F., Di Criscienzo, M., García-Hernández, D. A., et al. 2019, MNRAS, 482, 4733

Dell'Agli, F., Di Criscienzo, M., Ventura, P., et al. 2018, MNRAS, 479, 5035

Doherty, C. L., Gil-Pons, P., Siess, L., Lattanzio, J. C., \& Lau, H. H. B. 2015, MNRAS, 446, 2599

Eddington, A. S. 1913, MNRAS, 73, 359

Evans, A., \& Gehrz, R. D. 2012, BASI, 40, 213

Fazio, G. G., Hora, J. L., Allen, L. E., et al. 2004, ApJS, 154, 10

Feast, M., Whitelock, P., \& Menzies, J. 2002, MNRAS, 329, L7

Feast, M. W., Glass, I. S., Whitelock, P. A., \& Catchpole, R. M. 1989, MNRAS, 241, 375

Ferrarotti, A. S., \& Gail, H.-P. 2006, A\&A, 447, 553

Freedman, W. L., Madore, B. F., Scowcroft, V., et al. 2011, AJ, 142, 192

Gallart, C., Aparicio, A., Freedman, W. L., et al. 2004, AJ, 127, 1486

Galliano, F. 2018, MNRAS, 476, 1445

Gehrz, R. D., Roellig, T. L., Werner, M. W., et al. 2007, RScI, 78, 011302

Gerasimovič, B. P. 1928, PNAS, 14, 963
Glass, I. S., Schultheis, M., Blommaert, J. A. D. L., et al. 2009, MNRAS, 395, L11

Goldman, S. R., van Loon, J. Th., Gómez, J. F., et al. 2018, MNRAS, 473,3835

Goldman, S. R., van Loon, J. Th., Zijlstra, A. A., et al. 2017, MNRAS, 465,403

Gordon, K. D., Meixner, M., Meade, M. R., et al. 2011, AJ, 142, 102

Hamedani Golshan, R., Javadi, A., van Loon, J. T., Khosroshahi, H., \& Saremi, E. 2017, MNRAS, 466, 1764

Harris, W. E. 1996, AJ, 112, 1487

Hashemi, S. A., Javadi, A., \& van Loon, J. T. 2019, MNRAS, 483, 4751

Herwig, F. 2005, ARA\&A, 43, 435

Höfner, S., \& Olofsson, H. 2018, A\&ARv, 26, 1

Huang, C. D., Riess, A. G., Hoffmann, S. L., et al. 2018, ApJ, 857, 67

Hughes, S. M. G., \& Wood, P. R. 1990, AJ, 99, 784

Ita, Y., \& Matsunaga, N. 2011, MNRAS, 412, 2345

Ita, Y., Tanabé, T., Matsunaga, N., et al. 2004a, MNRAS, 347, 720

Ita, Y., Tanabé, T., Matsunaga, N., et al. 2004b, MNRAS, 353, 705

Javadi, A., Saberi, M., van Loon, J. T., et al. 2015, MNRAS, 447, 3973

Javadi, A., van Loon, J. T., Khosroshahi, H., \& Mirtorabi, M. T. 2013, MNRAS, 432, 2824

Javadi, A., van Loon, J. T., Khosroshahi, H. G., et al. 2017, MNRAS, 464, 2103

Javadi, A., van Loon, J. T., \& Mirtorabi, M. T. 2011a, MNRAS, 411, 263

Javadi, A., van Loon, J. T., \& Mirtorabi, M. T. 2011b, MNRAS, 414, 3394

Jones, O. C., Meixner, M., Justtanont, K., \& Glasse, A. 2017, ApJ, 841, 15

Karakas, A. I., \& Lattanzio, J. C. 2014, PASA, 31, e030

Karambelkar, V. R., Adams, S. M., Whitelock, P. A., et al. 2019, arXiv:1901. 07179

Kasliwal, M. M., Bally, J., Masci, F., et al. 2017, ApJ, 839, 88

Kirby, E. N., Rizzi, L., Held, E. V., et al. 2017, ApJ, 834, 9

Kovács, G. 2000, A\&A, 363, 1

Lagadec, E., \& Zijlstra, A. A. 2008, MNRAS, 390, L59

Lakićević, M., van Loon, J. Th., Meixner, M., et al. 2015, ApJ, 799, 50

Lebzelter, T., \& Wood, P. R. 2005, A\&A, 441, 1117

Lee, H., Skillman, E. D., Cannon, J. M., et al. 2006, ApJ, 647, 970

Liljegren, S., Höfner, S. H., Freytag, B., \& Bladh, S. 2018, A\&A, 619, A47

Lomb, N. R. 1976, Ap\&SS, 39, 447

Lorenz, D., Lebzelter, T., Nowotny, W., et al. 2011, A\&A, 532, 78

Marigo, P., Girardi, L., Bressan, A., et al. 2008, A\&A, 482, 883

Marigo, P., Girardi, L., Bressan, A., et al. 2017, ApJ, 835, 77

Mateo, M. 1998, ARA\&A, 36, 435

Mathias, P., Aurière, M., López Ariste, A., et al. 2018, A\&A, 615, A116

McConnachie, A. W. 2012, AJ, 144, 4

McDonald, I., Boyer, M. L., Groenewegen, M. A. T., et al. 2019, MNRAS, 484, L85

McDonald, I., Boyer, M. L., van Loon, J. Th., et al. 2011, ApJS, 193, 23

McDonald, I., De Beck, E., Zijlstra, A. A., \& Lagadec, E. 2018, MNRAS, 481, 4984

McDonald, I., \& Trabucchi, M. 2019, arXiv:1901.06325

McDonald, I., van Loon, J. T., Dupree, A. K., \& Boyer, M. L. 2010, MNRAS, 405, 1711

McDonald, I., Zijlstra, A. A., Sloan, G. C., et al. 2014, MNRAS, 439, 2618

McQuinn, K. B. W., Skillman, E. D., Berg, D., et al. 2013, AJ, 146, 145 
McQuinn, K. B. W., Woodward, C. E., Willner, S. P., et al. 2007, ApJ, 664,850

Meixner, M., Gordon, K. D., Indebetouw, R., et al. 2006, AJ, 132, 2268

Menzies, J., Feast, M., Whitelock, P., et al. 2008, MNRAS, 385, 1045

Menzies, J. W., Feast, M. W., Whitelock, P. A., \& Matsunaga, N. 2011, MNRAS, 414, 3492

Menzies, J. W., Whitelock, P. A., \& Feast, M. W. 2015, MNRAS, 452, 910

Menzies, J. W., Whitelock, P. A., Feast, M. W., \& Matsunaga, N. 2010, MNRAS, 406, 86

Momany, Y., Held, E. V., Saviane, I., \& Rizzi, L. 2002, A\&A, 384, 393

Nanni, A., Bressan, A., Marigo, P., \& Girardi, L. 2013, MNRAS, 434, 2390

Nanni, A., Bressan, A., Marigo, P., \& Girardi, L. 2014, MNRAS, 438, 2328

Nicholls, C. P., Wood, P. R., Cioni, M. R. L., \& Soszyński, I. 2009, MNRAS, 399, 2063

Poudel, S., Kulkarni, V. P., Morrison, S., et al. 2017, MNRAS, 473, 3559

Rafelski, M., Wolfe, A. M., Prochaska, J. X., Neeleman, M., \& Mendez, A. J. 2012, ApJ, 755, 89

Riebel, D., Boyer, M. L., Srinivasan, S., et al. 2015, ApJ, 807, 1

Riebel, D., Meixner, M., Fraser, O., et al. 2010, ApJ, 723, 1195

Rieke, G. H., Wright, G. S., Böker, T., et al. 2015, PASP, 127, 584

Salvatier, J., Wieckiâ, T. V., \& Fonnesbeck, C. 2016, Pymc3: Python Probabilistic Programming, Framework, Astrophysics Source Code Library, ascl:1610.016

Saviane, I., Rizzi, L., Held, E. V., Bresolin, F., \& Momany, Y. 2002, A\&A, 390, 59

Scargle, J. D. 1982, ApJ, 263, 835

Scowcroft, V., Freedman, W. L., Madore, B. F., et al. 2011, ApJ, 743, 76

Skillman, E. D., Terlevich, R., \& Melnick, J. 1989, MNRAS, 240, 563

Sloan, G. C., Kraemer, K. E., McDonald, I., et al. 2016, ApJ, 826, 44

Sloan, G. C., Matsuura, M., Lagadec, E., et al. 2012, ApJ, 752, 140

Sloan, G. C., Matsunaga, N., Matsuura, M., et al. 2010, ApJ, 719, 1274
Sloan, G. C., Matsuura, M., Zijlstra, A. A., et al. 2009, Sci, 323, 353

Soszyński, I., Udalski, A., Szymański, M. K., et al. 2009, AcA, 59, 239

Stetson, P. B. 1987, PASP, 99, 191

Temim, T., Dwek, E., Tchernyshyov, K., et al. 2015, ApJ, 799, 158

Trabucchi, M., Wood, P. R., Montalbán, J., et al. 2017, ApJ, 847, 139

Trabucchi, M., Wood, P. R., Montalbán, J., et al. 2018, MNRAS, 482, 929

Udalski, A., Kubiak, M., \& Szymański, M. 1997, AcA, 47, 319

van Loon, J. T. 2000, A\&A, 354, 125

van Loon, J. T. 2006, in ASP Conf. Ser. 353, Stellar Evolution at Low Metallicity: Mass Loss, Explosions, Cosmology, ed. H. J. G. L. M. Lamers et al. (San Francisco, CA: ASP), 211

van Loon, J. T., Marshall, J. R., \& Zijlstra, A. A. 2005, A\&A, 442, 597

VanderPlas, J. T. 2018, ApJS, 236, 16

Weisz, D. R., Dolphin, A. E., Skillman, E. D., et al. 2014, ApJ, 789, 147

Werner, M. W., Roellig, T. L., Low, F. J., et al. 2004, ApJS, 154, 1

Whitelock, P. A. 2012, Ap\&SS, 341, 123

Whitelock, P. A., Feast, M. W., Marang, F., \& Groenewegen, M. A. T. 2006 MNRAS, 369, 751

Whitelock, P. A., Kasliwal, M., \& Boyer, M. 2017, in European Physical Journal Web of Conf. 152, Wide-Field Variability Surveys: A 21st Century Perspective, ed. M. Catalan \& W. Gieren, 1009, arXiv:1702.06797

Whitelock, P. A., Menzies, J. W., Feast, M. W., et al. 2009, MNRAS, 394, 795

Whitelock, P. A., Menzies, J. W., Feast, M. W., \& Marigo, P. 2018, MNRAS, 473, 173

Wood, P. R. 2015, MNRAS, 448, 3829

Wood, P. R., Alcock, C., Allsman, R. A., et al. 1999, in IAU Symp. 191, Asymptotic Giant Branch Stars, ed. T. Le Bertre, A. Lèbre, \& C. Waelkens (Cambridge: Cambridge Univ. Press), 151

Yuan, W., Macri, L. M., Javadi, A., Lin, Z., \& Huang, J. Z. 2018, AJ, 156, 112

Zhukovska, S., Gail, H. P., \& Trieloff, M. 2008, A\&A, 479, 453 